\title{
SESCA: Predicting Circular Dichroism Spectra from Protein Molecular Structures
}

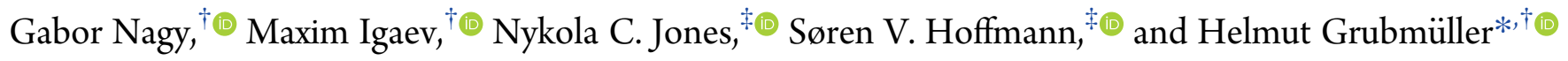 \\ ${ }^{\dagger}$ Department of Theoretical and Computational Biophysics, Max Planck Institute for Biophysical Chemistry, Am Fassberg 11, \\ D-37077 Göttingen, Germany \\ ${ }^{*}$ ISA, Department of Physics \& Astronomy, Aarhus University, Ny Munkegade 120, DK 8000 Aarhus C, Denmark
}

Supporting Information

ABSTRACT: Circular dichroism (CD) spectroscopy is a highly sensitive but low-resolution technique to study the structure of proteins. Combined with molecular modeling or other complementary techniques, CD spectroscopy can provide essential information at higher resolution. To this end, we introduce a new computational method to calculate the electronic circular dichroism spectra of proteins from a structural model or ensemble using the average secondary structure composition and a precalculated set of basis spectra. The method is designed for model validation to estimate the error of a given protein structural model based on the measured CD spectrum. We compared the predictive power of our method to that of existing algorithms, namely, DichroCalc and PDB2CD, and found that it predicts CD spectra more accurately. Our results indicate that the derived basis sets are robust to both experimental errors in the reference spectra and the choice of the secondary structure classification algorithm. For over $80 \%$ of the globular reference proteins, our basis sets accurately predict the experimental spectrum solely from their secondary structure composition. For the remaining $20 \%$, correcting for intensity normalization considerably improves the prediction power. Additionally, we show that the predictions for short peptides and an example complex of intrinsically disordered proteins strongly benefit from accounting for side-chain contributions and structural flexibility.

\section{INTRODUCTION}

Electronic circular dichroism (CD) spectroscopy is a widely applied optical method to study the structure and structural changes of biomolecules such as proteins, nucleic acids, and carbohydrates. ${ }^{1}$ As a very sensitive tool, $\mathrm{CD}$ is often used as a quality control for recombinant proteins or to monitor changes of the protein structure during folding, aggregation, and binding events. Because of its sensitivity, CD spectroscopy does not require large amounts of protein or special labeling and can be readily used in aqueous solutions.

At a more quantitative level, the CD spectra of proteins in the far ultraviolet (UV) range $(180-250 \mathrm{~nm})$ provide structural information. The main contributor to a protein CD spectrum in this range is the absorption of partially delocalized peptide bonds of the backbone, such that the spectrum is mainly determined by the secondary structure (SS).$^{2-5}$ However, isolated amino acids, except glycine, also show a $\mathrm{CD}$ signal in this wavelength range. ${ }^{6-8}$ Thus, amino acid (AA) side chains also contribute to the protein $\mathrm{CD}$ spectrum, albeit to a smaller extent.

To extract information from CD spectra, it is essential to establish a quantitative link between structural models and the observed spectra. Since the 1980s, two major categories of methods have been established: spectrum deconvolution methods (Figure 1A) predict the SS composition of a protein from its $C D$ spectrum, whereas spectrum prediction methods (Figure $1 \mathrm{~B}-\mathrm{D})$, vice versa, determine the $\mathrm{CD}$ spectrum from a structure.

Spectrum deconvolution methods (e.g., CCA, K2D3, and BeStSel $)^{9-11}$ estimate the SS composition of proteins with unknown structure, based on the observation that proteins with different SS-s have different CD spectra. Accordingly, the measured $C D$ spectrum is approximated by a linear combination of pure SS spectra or, more generally, properly chosen basis spectra. The coefficients obtained by this approximation provide the fractions of SS elements in the target protein (top-down arrows in Figure 1A). Basis spectra have been previously derived from either the CD spectra of model peptides or a larger set of reference proteins with known CD spectra and SS composition. ${ }^{10}$

The obtained SS information can also be used to validate structural models. To this end, SS estimates from CD deconvolution are compared to the SS composition of a proposed model (Figure 1A bottom). However, the reliability and robustness of these methods depend on the quality of the

Received: February 27, 2019

Published: August 11, 2019 


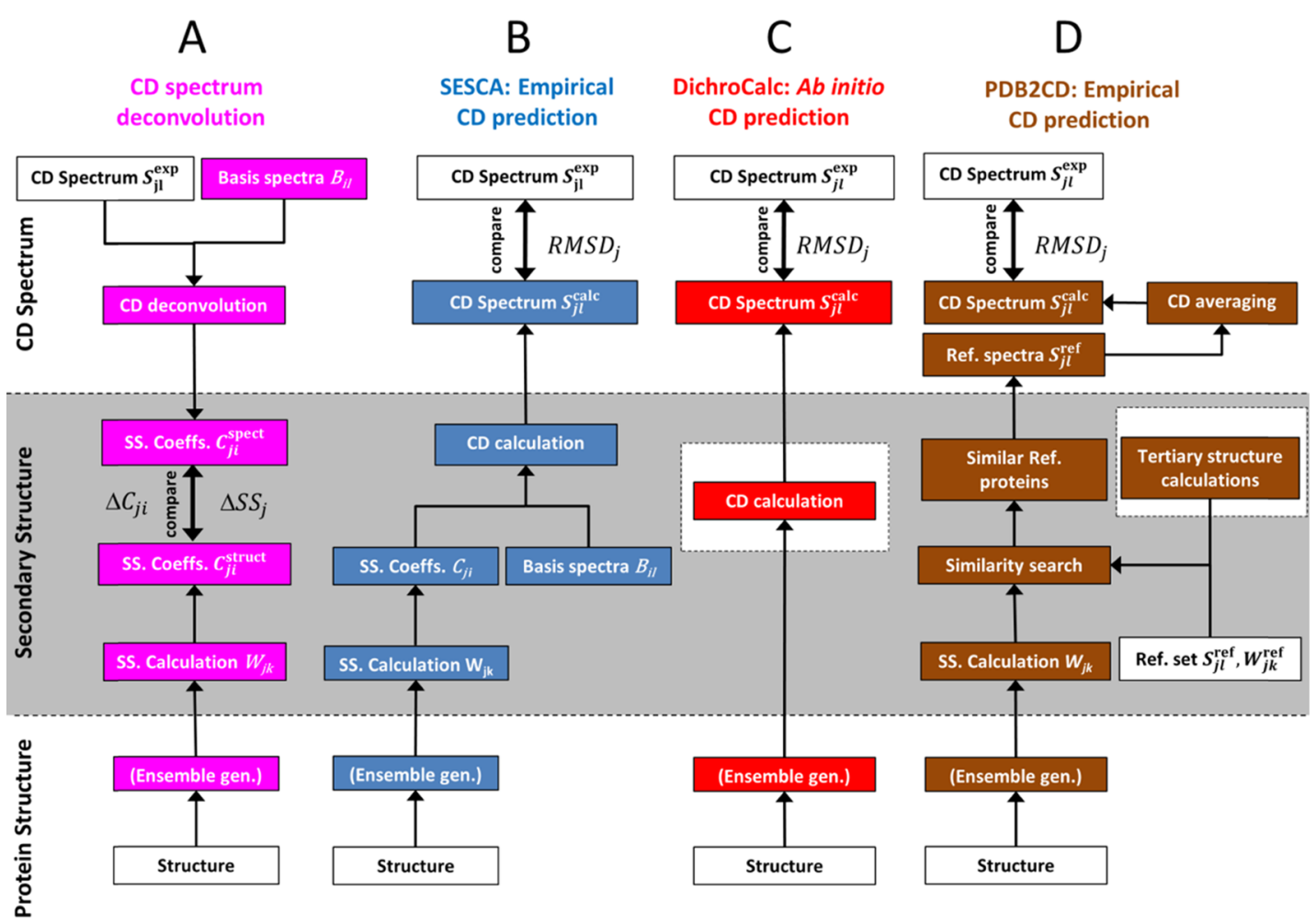

Figure 1. Relating a protein structure to its $\mathrm{CD}$ spectrum. White rectangles represent experimental data; magenta, blue, red, and brown fields are related to spectrum deconvolution and three different spectrum calculation methods (SESCA, DichroCalc, and PDB2CD), respectively. For spectrum deconvolution (column A), the secondary structure is determined independently from both the CD spectrum and the structural model and subsequently compared. In contrast, spectrum prediction methods (columns B-D) compute a CD spectrum from the structure, which is compared to the measured spectrum. Fields in the shaded area denote calculation steps at the secondary structure level.

measured spectrum, ${ }^{4,12,13}$ and even established deconvolution methods may yield largely different SS estimates depending on their fitting procedure and basis spectra used. ${ }^{14}$

The reverse approach, model validation based on spectrum predictions, may allow a more robust comparison between the model and the measured CD spectra, as well as easier diagnostics of potential measurement errors. The most advanced $\mathrm{CD}$ spectrum prediction methods are ab initio methods (Figure 1C) and typically require computationally demanding excited-state quantum mechanics (QM) or density functional calculations. ${ }^{15-17}$ These methods determine the light absorption of molecules directly from their structure, but the large computational effort usually limits such calculations to rather small peptides. To speed up calculations, a simplified ab initio spectrum prediction algorithm, called the matrix method, ${ }^{18}$ has been implemented in the DichroCalc program. ${ }^{19}$ DichroCalc determines the most important features of the CD spectrum of a protein based on its average crystallographic structure and parameters derived from ab initio QM calculations, albeit with limited accuracy. ${ }^{20}$

In contrast, and as an alternative to ab initio spectrum predictions, the recently proposed PDB $2 \mathrm{CD}^{20}$ method (Figure 1D) estimates the CD spectrum of a target protein from known CD spectra of structurally similar proteins selected from a reference set. By substituting the computationally demanding QM calculations with a secondary- and tertiary-structure-based similarity search among the reference proteins, PDB2CD achieves markedly higher average prediction accuracy than DichroCalc. However, this accuracy is limited by the number of reference proteins structurally similar to the target.
Here, we develop and test a spectrum prediction method for the efficient validation and refinement of protein structures against measured CD spectra. Our structure-based empirical spectrum calculation approach (SESCA) combines elements of both deconvolution and spectrum prediction. Specifically, like deconvolution methods, SESCA uses secondary-structurerelated basis spectra, but unlike deconvolution methods, it obtains the required coefficients directly from a model structure (Figure $1 \mathrm{~B}$ ), similarly to other CD prediction methods (Figure 1C,D).

Because SESCA determines basis set coefficients from a model protein structure, its predictions are unaffected by potential experimental errors from the measured CD spectrum of the target protein, while the use of precalculated basis spectra allows fast yet accurate spectrum predictions. SESCA basis sets combine all of the experimentally determined structural and spectral information to describe the average CD signals for the local conformations of peptide bonds ("SS classes"). Due to this averaging over many reference proteins, our basis sets are also expected to be robust to experimental noise in both $\mathrm{CD}$ spectra and reference structures of individual proteins. Additionally, because our method is based on statistics of peptide bond conformations rather than on identifying similar tertiary structures, we expect SESCA to have a larger predictive power than PDB2CD, particularly for proteins with structures dissimilar to those in the reference set or even with no structure at all.

Further, extracting precalculated basis spectra from the available reference proteins reduces the computational complexity of $\mathrm{CD}$ predictions to the calculation of a single linear combination. Because its basis spectrum coefficients rely 

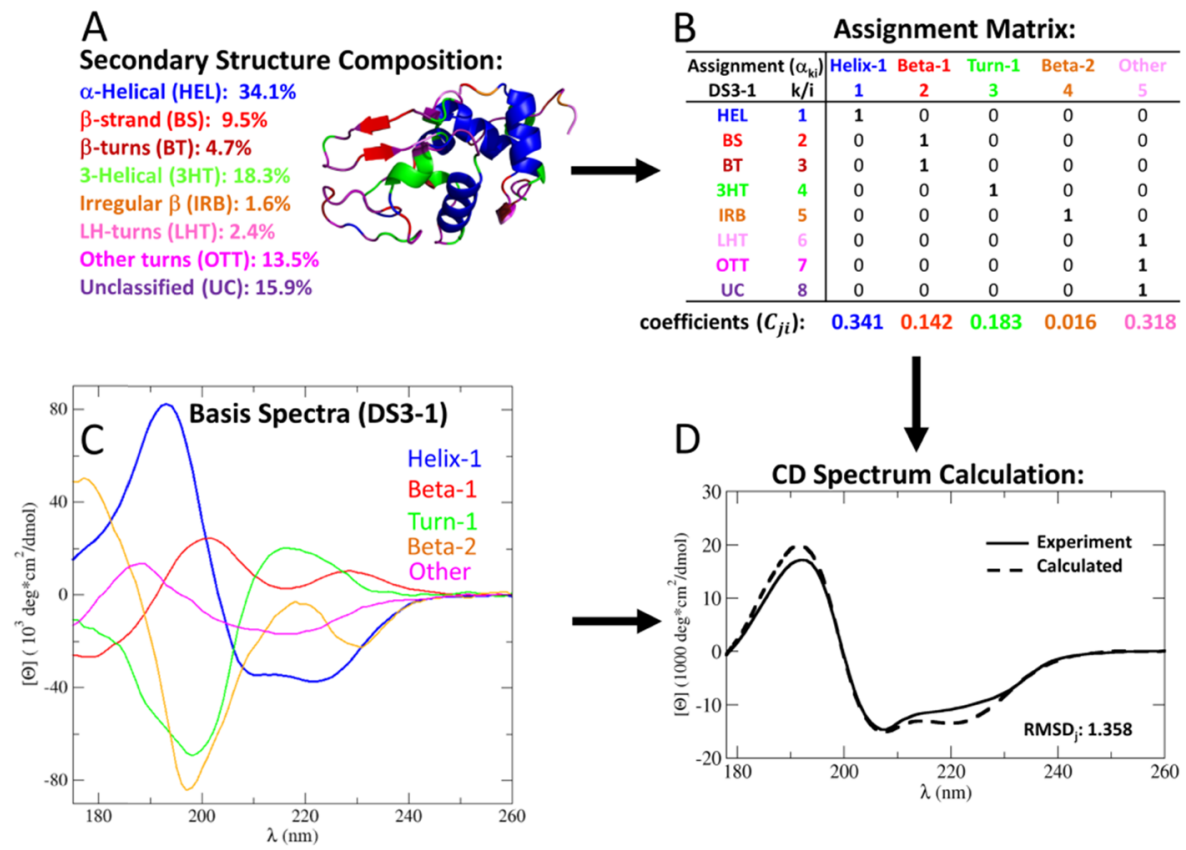

Figure 2. SESCA's CD spectrum calculation scheme. (A) Secondary structure composition (in colors) and a cartoon representation of a protein $j$ (here, lysozyme). The secondary structure information is translated into a CD spectrum via a basis set (here, DS3-1) composed of an assignment matrix and a set of basis spectra. (B) The assignment matrix $\alpha_{k i}$ groups the secondary structure elements $k$ (on the left) into secondary structure classes $i$ (on the top). The secondary structure class composition ( $C_{j i}$ bottom line) determines the coefficients in the weighted averaging of basis spectra in (C), which are used to calculate the CD spectrum for the protein. (D) The calculated CD spectrum (dashed line) is compared to the measured spectrum (solid line) via a root-mean-squared deviation, $\mathrm{RMSD}_{\mathrm{j}}$. Throughout, mean residue ellipticity units are used for the spectra.

only on the SS composition of the model structure, SESCA avoids costly chromophore calculations, as well as tertiary structure and fold-recognition steps. Finally, by taking advantage of the existing high-throughput SS classification methods, SESCA also allows CD predictions from large structural ensembles.

In this study, our approach is evaluated and optimized using various available SS classification algorithms. Additionally, we address (a) the effects of conformational flexibility by using structural ensembles during $C D$ predictions and (b) the contribution of natural amino acid side chains. We show that including these contributions increases the prediction accuracy particularly for peptides and an intrinsically disordered protein (IDP) complex and, thus, should also enable the refinement of IDP ensemble models against measured CD spectra.

\section{THEORETICAL BACKGROUND}

Basis Spectrum Calculations. We will initially assume that the CD spectra are mainly determined by the local conformation of the peptide bonds and subsequently also consider the effects of AA side-chain groups within the same framework. The workflow of our structure-based empirical CD prediction method is described in Figure 2. First, the local backbone conformation is grouped into secondary structure elements with established methods (Figure 2A), to obtain the SS information from the protein structure required for the SESCA calculations. Second, these SS elements are combined into broader classes (Figure 2B) for which basis spectra are determined (Figure 2C). This grouping is necessary to control the number of basis spectra and optimize the prediction accuracy. The predicted $\mathrm{CD}$ spectrum of the protein is calculated from weighted averages of basis spectra (Figure $2 \mathrm{D})$, which is compared to the measured CD spectrum to determine the quality of the model structure. Note that besides the SS composition obtained from the model, the parameters SESCA predictions require are stored in a collection of basis spectra and the assignment matrix used for grouping the SS elements. Henceforth, we refer to these parameters as a basis set.

We derive SESCA basis sets from a set of $N$ globular reference proteins with known structures and CD spectra $S_{j}^{\exp }(\lambda)$. The latter are approximated by a weighted sum of $F$ basis spectra $B_{i}(\lambda)$

$$
S_{j}^{\text {calc }}(\lambda)=\sum_{i=1}^{F} C_{j i} B_{i}(\lambda)
$$

where the weights (coefficients) $C_{j i}$ are the fractions of AAs in protein $j$ that were assigned to the SS class $i$. We derive the basis spectra by minimizing the average root-mean-squared deviation (RMSD) between the measured spectra of the reference proteins $S_{j}^{\exp }(\lambda)$ and those calculated from the secondary structure $S_{j}^{\text {calc }}(\lambda)$ for all reference proteins

$$
\mathrm{RMSD}_{\text {set }}=\frac{1}{N} \sum_{j=1}^{N} \sqrt{\frac{1}{L} \sum_{\lambda=\lambda_{1}}^{\lambda L}\left[S_{j}^{\text {calc }}(\lambda)-S_{j}^{\exp }(\lambda)\right]^{2}}
$$

as described in the supplementary materials (SM) Section S1.

We note that in spectrum deconvolution methods ${ }^{9,10,21}$ basis spectra are derived via the same notion but are applied differently. In deconvolution, the basis spectrum coefficients $\left(C_{j i}\right)$ are treated as fit parameters, which yield the SS content from the known CD spectrum of a target protein (Figure 1A). In our approach, the SS content is extracted from the known structure and combined into the basis spectrum coefficients to predict the CD spectrum. $C_{j i}$ are calculated from the fraction of residues $\left(W_{j k}\right)$ classified as the SS element $k$ in the structural 
model of protein $j$ via an assignment matrix $\mathbf{A}=\left\{\alpha_{k i}\right\}$ (Figure 2B) such that

$$
C_{j i}=\sum_{k=1}^{K} W_{j k} \alpha_{k i}
$$

and the SS information described by the $K$ secondary structure elements is now contained in $F$ structural classes. A combination of eqs 1 and 3 relates the $C D$ spectrum of a protein to its secondary structure composition

$$
S_{j}^{\mathrm{calc}}(\lambda)=\sum_{k=1}^{K} \sum_{i=1}^{F} W_{j k} \alpha_{k i} B_{i}(\lambda)
$$

in a way that for a given $\mathbf{A}$ matrix, the optimal basis spectra $B_{i}(\lambda)$ are readily calculated from the reference proteins by minimizing $\mathrm{RMSD}_{\text {set. }}$. The assignment factors $\alpha_{k i}$ determine the average backbone structure of SS classes and the number and shape of the optimal basis spectra and consequently strongly influence the predictive power of the basis set.

Assignment Optimization. To find the most predictive basis sets, the number of basis spectra (basis set size) and the assignment of the SS elements are optimized. To this end, a Monte Carlo (MC) search is performed among the possible A matrices, where both $\alpha_{k i}$ and $\sum_{i=1}^{F} \alpha_{k i}$ are limited to $\{0,1\}$, ensuring that SS elements are assigned exclusively to one of the SS classes. When basis sets are optimized under these constraints, henceforth referred to as "hard basis sets" and "hard optimization", the procedure results in orthogonal SS classes and normalized basis spectra. For every resulting assignment, a subset of the reference proteins (training set) is used to calculate the ideal basis spectra as described in SM Section S1, which are then used to predict the CD spectra of remaining reference proteins (evaluation set). The average deviation of the predicted CD spectra $\left(\mathrm{RMSD}_{\text {set }}\right)$ is used to score the predictive power of the assignment during the optimization. Finally, the basis spectra of the best assignments are recalculated using all of the reference proteins to acquire the final optimized basis set. Further details on the hard optimization procedure are provided in SM Section S2.

Including Side-Chain Contributions. To assess the contribution of AA side chains, we assume that there are two main contributors to the CD spectra of proteins: the SSdependent signal of the peptide bonds and the chromophores of the amino acid side chains, with no coupling between the side chains and the rest of the protein. This assumption allows us to represent the average contribution of side-chain groups by additional basis spectra and the calculation of a backboneindependent side chain baseline. The baseline for each protein is determined by the weighted average of the individual sidechain basis spectra, where the weighing factor is the corresponding AA content extracted from the protein sequence ( similarly to eq 1). Details of the calculation of side-chain contributions are provided in SM Section S3. Note that the results we present in Secondary Structure-Based CD calculations are based purely on the SS information of the protein, and the effects of side-chain corrections are discussed in Including Side-Chain Contributions to the CD Spectrum.

Basis Set Quality Assessment. The quality of the optimized basis sets is assessed as follows. First, we determine the predictive power of each basis set by cross-validation. The prediction accuracy is quantified by computing $\mathrm{RMSD}_{\text {set }}$ for a set of proteins that is not used during the basis set determination (henceforth, prediction accuracy or RMSD cross ). Second, we calculate $\mathrm{RMSD}_{\text {set }}$ for CD spectra of the reference protein set from which the basis sets are derived (henceforth, fitting accuracy or $\mathrm{RMSD}_{\text {fit }}$ ). The difference between $\mathrm{RMSD}_{\text {fit }}$ and $\mathrm{RMSD}_{\text {cross }}$ for a basis set quantifies overfitting during the optimization. For basis sets with no overfitting, $\mathrm{RMSD}_{\text {fit }}$ and $\mathrm{RMSD}_{\text {cross }}$ should be similar, while for a basis set or spectrum prediction method with significant overfitting, $\mathrm{RMSD}_{\text {fit }}$ should be significantly lower than $\mathrm{RMSD}_{\text {cross }}$.

We also perform additional analyses to determine the limits of basis set accuracy using the applied reference protein set. To this end, we derive series of specialized basis sets with 1 to $F$ basis spectra (where $F$ is the number of the SS elements in the classification protocol used). These basis sets are derived by a different, unconstrained optimization scheme aimed solely to minimize $\mathrm{RMSD}_{\text {fit }}$, with no regard to overfitting or predictive power. Details about this optimization approach ("soft optimization") are provided in SM Section S4. The "soft basis sets" serve as reference points on how accurately the CD spectra of the reference protein set can be described using the limited information encoded in the SS composition of the protein.

Finally, we perform a principal component analysis (PCA) on the $C D$ spectra of the reference protein set we derived our basis sets from. Constructing basis sets from the principle component $(\mathrm{PC})$ vectors of the $\mathrm{CD}$ spectra allows us to estimate the upper limit of prediction accuracy for globular proteins using a given basis set size. Details of this analysis are described in SM Section S5. This upper accuracy limit is used to estimate how much could we improve our prediction algorithm by including extra structural information in addition to the SS composition.

Structure Validation. Once derived, SESCA basis sets can be used to validate protein structural models based on the measured $C D$ spectrum of the protein. To quantify the deviation between predicted and measured CD spectra, SESCA computes RMSD as well as NRMSD values. The latter is an RMSD normalized for the average amplitude of the measured spectrum according to Mao et al. ${ }^{22}$ When multiple proposed models are compared to the same CD spectrum, the ratio of RMSD and NRMSD values between models is unchanged. Because the two metrics thus lead to identical conclusions, we will present results using RMSD values only.

One can estimate the total error in the SS composition of a structural model $\left(\Delta S_{j}\right)$ for protein $j$ based on the average deviation $\left(\mathrm{RMSD}_{j}\right)$ between its predicted and experimentally measured CD spectra. Note that even for the correct SS composition $C_{j i}^{0}$ (i.e., $\Delta S_{j}=0$ ), the $\mathrm{CD}$ spectrum predicted at wavelength $l$ from the model would still deviate from the measured spectrum by $\Delta S_{j l}=S_{j l}^{\exp }-S_{j l}^{0}$, due to the approximations in our prediction method (Basis Spectrum Calculations), as well as due to the experimental error of the measured CD spectrum. To accurately determine $\Delta S S_{j}$ for our model, one thus has to separate the SS-dependent error $\left(M_{j}\right)$ in the predicted spectrum, introduced by the structural model, from the SS-independent error $\mathrm{RMSD}_{j}^{0}=\sqrt{\frac{1}{L} \cdot \sum_{l=1}^{L} \Delta S_{j l}^{2}}$, which describes the deviation between the experimental spectrum and the spectrum predicted from $C_{j i}^{0}$.

To this end, we consider predicting the CD spectrum from an imperfect structural model with SS composition $C_{j i}$, which deviates from the correct SS composition by $\Delta S_{j}=\frac{1}{2} \sum_{i=1}^{F}\left|\Delta C_{j i}\right|$, where $\Delta C_{j i}=C_{j i}^{0}-C_{j i}$ is the error in 
the coefficient of SS class $i$ for protein $j$. In Section S6, we show that the SS-dependent error for a given $\Delta C_{j i} / \Delta S S_{j}$ ratio is proportional to $\Delta S S_{j}\left(M_{j}=m_{j} \cdot \Delta S S_{j}\right)$ with a slope $m_{j}$ that depends on the basis spectra as well as on the $\Delta C_{j i}$ ratios. Assuming that the SS-dependent and SS-independent errors at different wavelengths are statistically independent, the RMSD of the predicted spectrum is

$$
\begin{aligned}
& \mathrm{RMSD}_{j}^{2}=\left(m_{j} \cdot \mathrm{SS}_{j}\right)^{2}+\mathrm{RMSD}_{j}^{0^{2}} \approx\left(m_{j} \cdot \mathrm{SS}_{j}\right)^{2} \\
& \quad \text { for } M_{j} \gg \mathrm{RMSD}_{j}^{0}
\end{aligned}
$$

Even if the two error terms are correlated, the average error of the predicted spectra may change between $\mathrm{RMSD}_{j}=m_{j} \cdot \Delta \mathrm{SS}_{j}+$ $\mathrm{RMSD}_{j}^{0}$ and $\mathrm{RMSD}_{j}=m_{j} \cdot \Delta \mathrm{SS}_{j}-\mathrm{RMSD}_{j}^{0}$. We note that if the $\mathrm{CD}$ spectra are free of systematic errors, $\mathrm{RMSD}_{j}^{0}$ describes the average noise of the $\mathrm{CD}$ measurements and should be statistically independent of $M_{j}$.

\section{MATERIALS AND METHODS}

Protein Reference Sets for Calibration. To derive and assess the basis sets required for SESCA, 102 reference proteins were collected for which both the $\mathrm{CD}$ spectrum and the structure have been determined. These were grouped into nonoverlapping subsets to separate basis set optimization (set SP175) from independent subsequent quality assessment (set TS8) by several levels of cross-validation (for a full list, see Tables S1-S3). All crystallographic structures were obtained from the protein databank (PDB). ${ }^{23}$

The SP175 set (Table S1) comprises 71 globular protein structures and their corresponding $\mathrm{CD}$ spectra, assembled by Lees et al. ${ }^{24}$ such that (1) its SS distribution determined by the DSSP algorithm ${ }^{25}$ reflects that of the full PDB, it features (2) high-resolution PDB structures (X-ray diffraction and nuclear magnetic resonance structures, average resolution $1.9 \AA$ ) and (3) high-quality synchrotron radiation CD spectra (wavelength range 175-269 $\mathrm{nm}$ ), and (4) the set represents the major protein classes and architectures of the $\mathrm{CATH}^{26}$ database, which classifies proteins based on structural similarity as well as evolutionary relations. We mainly use set SP175 to derive and optimize SESCA basis sets and to determine their fitting accuracy, but this set also represents globular proteins during our analyses, e.g., during the PCA of protein CD spectra.

The cross-validation set TS8 (Table S2) comprises eight additional globular proteins, selected from a set of 22 proteins, previously used for CD spectrum deconvolution. ${ }^{14}$ The CD spectra of the TS8 set were obtained from Perczel et al. ${ }^{10}$ and cover a slightly shorter spectral range $(178-260 \mathrm{~nm})$ than that covered by those of the SP175 set. The TS 8 crystal structures have an average resolution of $1.7 \AA$, and contain no missing residues. Set TS8 was used to assess the prediction accuracy of both hard and soft basis sets after optimization (Figure S1), as well as the PDB2CD method.

The SP175 data set is divided into two subsets for the hard optimization approach, a larger training set of 64 proteins (TR64) for optimizing the basis spectra, and a smaller evaluation set EV9 of nine proteins (Table S3) for assessing the predictive power of basis sets during their optimization. Two additional proteins with a $\beta$-sheet architecture were added to set EV9 from Perczel et al. to obtain a balanced distribution of main folds and sufficient sampling to avoid overfitting. Also, the protein structures of set EV9 (average resolution 1.6 $\AA$ ) contain no missing residues.
A third subset of SP175 (GP59 for "globular protein" set) comprising 59 globular proteins is used to estimate the average contribution of side chains to the CD spectra. Accordingly, GP59 was formed such that (1) its proteins maintain a wide variation of SS compositions and (2) their spectra are predicted with sufficient accuracy (see Effect of the Structural Models). Twenty short peptides (the GXG20 set) with the consensus sequence of Ac-GXG-NH $\mathrm{NH}_{2}$ (X stands for any AA) were added to GP59 to form a reference set of "mixed polypeptides" termed MP79. This final reference set is used to optimize all "mixed basis sets" with both backbone and side-chain contributions.

CD Spectrum Measurements. The CD spectra of set SP175 were provided by Kardos et al. and are deposited in the protein circular dichroism databank (PCDDB). ${ }^{27}$ The CD spectra of set TS8 were obtained from the literature (see Protein Reference Sets for Calibration). The CD spectra of set GXG20 peptides and the complex of the two disordered protein domains P53-AD2 and CBP-NCBD were recorded on the AU$\mathrm{CD}$ beam line at the ASTRID2 synchrotron radiation source (Aarhus, Denmark) under similar conditions (298 K, in $50 \mathrm{mM}$ $\mathrm{NaF}$ solution with $\mathrm{Na}_{2} \mathrm{HPO}_{4}$ buffer, $\mathrm{pH}=7.1$ ) over the wavelength range $178-300 \mathrm{~nm}$. Protein concentrations (0.5$2.0 \mathrm{mg} / \mathrm{mL}$ ) were determined from light absorption at 214 $\mathrm{nm}^{28}$ and, when possible, at $280 \mathrm{~nm}$ (for both domains as well as GYG and GWG). Both peptides and protein domains were produced by the company Karebay using solid-state peptide synthesis. The CD spectrum P53/CBP complex was recorded after $30 \mathrm{~min}$ of incubation time at room temperature with a 1:1 molar ratio. The CD spectra in all data sets were converted to mean residue ellipticity ( $[\Theta]$ or MRE) shown in the units of $10^{3}$ $\mathrm{deg} \cdot \mathrm{cm}^{2} / \mathrm{dmol}$, abbreviated as $\mathrm{kMRE}$.

Structural Models and Molecular Dynamics Simulations. The crystal structures of sets SP175 and TS8 were obtained from the $\mathrm{PDB}^{23}$ (entry codes and subset information provided in Tables $\mathrm{S} 1-\mathrm{S} 3$ ), as was the NMR solution structure of the P53/CBP complex (used in Case Study: CD Predictions for a Flexible Protein Complex, PDB code 2L14). Structural ensembles for each GXG20 peptide were generated using a 10 $\mu$ s long molecular dynamics (MD) simulation (recorded every $2 \mathrm{~ns}$ ) using the GROMACS simulation package ${ }^{29,30}$ (version 5.06) and the Charmm $36 \mathrm{~m}$ parameter set with the explicit TIP3P water model modified for the force field. ${ }^{26}$

The simulations were performed under periodic boundary conditions at $298 \mathrm{~K}$, with $\mathrm{Na}^{+}$and $\mathrm{Cl}^{-}$ions at $50 \mathrm{mM}$ ionic strength and protonation states corresponding to $\mathrm{pH}=7$. The size of the simulation box was chosen so as to keep $\sim 2 \mathrm{~nm}$ distance between any solute atom and the box boundaries, resulting in a simulation box with $\sim 5500$ atoms. All GXG20 simulations were started from an extended conformation.

The P53/CBP simulation was carried out similarly, except that the Charmm 22* parameter set $^{31}$ was used and the simulation box contained $\sim 82000$ atoms. The simulation was started using the first conformation of the NMR bundle, and protein conformations were recorded every $10 \mathrm{~ns}$ throughout a $10 \mu \mathrm{s}$ long simulation trajectory, resulting in an ensemble of 1000 conformations. Here, the obtained structural ensemble was compared with the starting structure as well as with the 20 conformations of the full NMR bundle for predicting the measured $C D$ spectrum and the $\mathrm{C}_{\alpha}$ secondary chemical shifts.

Secondary Structure Determination. The SS of all proteins was determined from the protein structure using three different algorithms: dictionary of secondary structure for proteins (DSSP), ${ }^{25}$ dihedral-based segment identification and 
classification (DISICL), 32 and the in-house algorithm hydrogen-bond-based secondary structure (HbSS) described below.

DSSP identifies eight SS elements (in Table S4) based on their distinctive backbone hydrogen-bonding patterns. DISICL uses two $(\phi, \psi)$ backbone dihedral angle pairs to classify tetrapeptide segments into 19 SS elements (DS_det, Table S5), which are grouped into eight broader SS classes in the simplified DISICL protocol (DS_sim).

The HbSS algorithm was designed to provide SESCA with a more fine-grained SS classification regarding $\beta$-sheet architectures. In particular, HbSS distinguishes between parallel and antiparallel $\beta$-strands based on the backbone hydrogen-bond patterns shown in Figure S7, and it also identifies three types of helices (listed in Table S6) and hydrogen-bonded turns based on the same patterns as used in DSSP. The HbSS classification protocol was also extended (HBSS_ext) based on the $\beta$-strand twist to determine the amount of left-handed, relaxed (nontwisted), and right-handed $\beta$-strands described by Ho et al., ${ }^{33}$ with boundaries of 3 and $23^{\circ}$, respectively, for both parallel and antiparallel strand arrangements. This extended structural classification protocol is designed to give results comparable with the estimated $\beta$-strand compositions from the deconvolution algorithm $\mathrm{BeStSel}^{9}$ (Table S7).

CD Spectrum and SS Predictions. Prior to analysis, crystallographic water, nonstandard residues, and cofactors were removed from the crystal structures of the data sets. Residue numbers and chain codes were relabeled to ensure compatibility with the analysis software. For all entries of the reference protein sets, the AA composition and the SS content were determined (Secondary Structure Determination). The CD spectra of reference proteins were predicted using several SESCA-optimized basis sets (see SM Tables S8 and S9) and the SS compositions obtained, as well as using the DichroCalc and PDB2CD software packages and the processed reference structures. For PDB2CD, the SP175 reference set was used to predict the spectra, while for DichroCalc, the peptide parameter set from Hirst et al. was selected for CD predictions, including backbone charge-transfer transitions, as well as aromatic and acidic side-chain chromophores. A PCA was applied to the SP175 CD spectra to determine the number of necessary spectral components and to probe correlations between the principal components, SS elements, and AA composition (see SM Section S5). For comparison, the SS content of each protein was estimated from its $C D$ spectrum using the deconvolution algorithms SELCON ${ }^{21}$ and BeStSel. These estimates were also included in the spectral component analysis (SM Section S5).

Averaging over Prediction Results. In this work, CD spectrum predictions were often used to determine properties averaged over a set of reference proteins such as the mean accuracy for the calculated spectra $\left(\mathrm{RMSD}_{\text {set }}\right)$ or the error of structural models $\left(\Delta S_{\text {set }}\right)$. These obtained properties are influenced by the errors of the prediction method (for SESCA, the errors of the basis spectra). To reduce the effect of nonsystematic errors from the prediction method, we calculated the mean property for each protein $j$ from multiple predictions, denoted

$$
A_{j}^{\text {mean }}=\frac{1}{M} \cdot \sum_{m=1}^{M} A_{j}^{m} \text { and } \sigma_{j}=\sqrt{\frac{1}{M} \cdot \sum_{m=1}^{M}\left(A_{j}^{m}-A_{j}^{\text {mean }}\right)^{2}}
$$

where $A_{j}^{m}$ is the property $A$ obtained for protein $j$ using the prediction $m$ and $\sigma_{j}$ is the scatter of the obtained properties. The average property over the protein set $\left(A_{\text {set }}^{\text {mean }}\right)$ and its standard deviation $\left(\sigma_{\text {set }}\right)$ was then calculated as

$$
A_{\text {set }}^{\text {mean }}=\frac{1}{N} \cdot \sum_{j=1}^{N} A_{j}^{\text {mean }} \text { and } \sigma_{\text {set }}=\sqrt{\frac{1}{N} \cdot \sum_{j=1}^{N} \sigma_{j}^{2}}
$$

When calculating mean properties for SESCA, we averaged over results obtained using four optimized basis sets: DS-dT, DSSP1, HBSS-3, and DS5-4, which differ in size and in the underlying SS classification protocol (see Table S8). In cases where the properties were not SESCA-specific, such as the scaling factors $\mathrm{SF}_{j}^{\text {mean }}$ used in Potential Measurement Errors of the Reference Set, two other methods, namely, PDB2CD and Bestsel der, were also included in the calculations. PDB2CD predicts $C D$ spectra from the reference crystal structures but (unlike SESCA) does not rely on basis spectra to do so. Bestsel_der is a control basis set that reconstructs the CD spectra from the SS composition estimated by the established deconvolution algorithm BeStSel. ${ }^{9}$ Because the BesStSel estimates are fitted to match the reference spectra (both for SP175 and TS8 sets), Bestsel_der allows us to estimate the best prediction accuracy for basis sets without significant systematic errors from the TS8 reference structures. We note that the Bestsel_der basis set was also derived using the methodology described in SM Section S1, where the estimated SS compositions of the SP175 set were utilized to determine the basis spectra and the TS8 estimates to cross-validate the basis set. However, as Bestsel_der simply reconstructs the reference spectra from the $\bar{B}$ eStSel SS estimates, it is not a true $\mathrm{CD}$ prediction basis set.

\section{RESULTS AND DISCUSSION}

We present our results in two sections. In Secondary StructureBased CD calculations, we assess the accuracy of our structurebased empirical spectrum calculation approach, SESCA, using SS information only. In Improving the CD Prediction Accuracy, we include the effects of side groups and backbone dynamics and explore the potential to further improve SESCA predictions for folded proteins, peptides, and IDPs.

Secondary Structure-Based CD Calculations. As the main determinant of prediction accuracy for SESCA is the choice of the basis set (defined in Basis Spectrum Calculations), we first assess the effects of this choice. To this end, using the 71 proteins of the SP175 reference set, we derived different basis sets with varying number and shape of the basis spectra, using different underlying SS classification protocols. All of these basis sets were determined by the hard optimization approach (see Section S2) and were subsequently cross-validated against the proteins of the TS8 reference set. The prediction and fitting accuracies for all basis sets were determined as described in Basis Set Quality Assessment, and the most predictive basis sets were analyzed further. In Performance Comparison, we compare the prediction accuracies achieved with the two established CD prediction methods DichroCalc and PDB2CD. Possible limitations of SESCA are explored in Basis Set Accuracy Limits using PCA and soft basis sets (see Basis Set Quality Assessment). Finally, in Model Validation based on CD predictions, we determine the sensitivity of spectrum prediction accuracy with respect to errors in the SS composition of the protein model. 
SESCA Basis Set Assessment. We derived basis sets with 1 to a maximum of 19 basis spectra, using five SS classification protocols in total (see Secondary Structure Determination). Each basis set was optimized using the TR64 and EV9 subsets of SP175 for training and evaluation, respectively. Subsequently, the basis spectra were recalculated from the full SP175 set, and thereafter, the basis sets were cross-validated against the TS8 set. The 10 top-ranking optimized basis sets achieved an average prediction accuracy of $\mathrm{RMSD}_{\mathrm{TS} 8}=3.3 \pm 0.6 \mathrm{kMRE}$ units $\left(10^{3} \mathrm{deg} \cdot \mathrm{cm}^{2} / \mathrm{dmol}\right)$. Notably, the average fitting accuracy $\mathrm{RMSD}_{\mathrm{SP} 175}=3.6 \pm 0.2 \mathrm{kMRE}$ is very similar, indicating little to no overfitting.

Table S8 (upper half) lists the average accuracies of the topranking basis sets for the predicted CD spectra of the SP175 set, its training (TR64) and evaluation (EV9) subsets, and the TS8 cross-validation set. For comparison, an additional seven nonoptimized basis sets are shown as well (Table S8, lower half). To assess the effects of the underlying SS classification, we compared the most accurate predictions for each classification algorithm from the basis sets DS-dT (DISICL), DSSP-1 (DSSP), and HBSS-3 (HbSS), respectively (marked by asterisks in Table S8). These three basis sets achieved RMSD $\mathrm{TS}_{\mathrm{TS}}$ values of $3.15 \pm 0.54,2.99 \pm 0.56$, and $3.30 \pm 0.60 \mathrm{kMRE}$, respectively. As these prediction accuracies are similar within statistical error, the choice of the SS classification protocol does not seem to markedly affect the predictive power of SESCA.

Unexpectedly, for the three most accurate basis sets mentioned above, regardless of the underlying classification algorithm, the prediction accuracy is also somewhat higher than the fitting accuracy $\left(\mathrm{RMSD}_{\mathrm{SP} 175}\right)$ of the SP175 reference set $(3.70 \pm 0.24,3.78 \pm 0.26$, and $3.75 \pm 0.23 \mathrm{kMRE}$, respectively). We tentatively attribute this finding to the slightly higher average data quality of the TS8 set. This explanation is also supported by the fact that the removal of certain outliers with poor mean prediction accuracies found in the SP175 set (see in Potential Measurement Errors of the Reference Set) improved the RMSD beyond that of TS8.

Notably, all 10 top-ranking basis sets listed in Table S8 contain between three and six basis spectra. In fact, in all basis sets considered, the number of basis spectra drops to eight or less but not below three during optimization. This finding indicates that at least three distinct basis spectra are required to explain the diversity of the observed CD spectra and that the limited accuracy of both structures and measured CD spectra in the reference data sets does not allow for more than eight basis spectra without overfitting. This finding is also in line with the prediction accuracies of the nonoptimized basis sets shown in Table S8 (bottom) as well as with the analysis PCA and soft basis sets of the best achievable fitting accuracy in Performance Comparison.

Figure $3 \mathrm{~A}-\mathrm{C}$ shows the basis spectra of the three most accurate basis sets. As the most distinctive common feature, all three sets contain a very similar basis spectrum largely representing $\alpha$-helical structure elements (blue lines), which, as one should expect, closely resembles the basis spectra attributed to $\alpha$-helices in previous studies. ${ }^{4,34,35}$ The $\beta$-strand basis spectra are also similar to each other, although smaller differences exist depending on the underlying $\beta$-strand classifications. These two basis spectra appear consistently throughout the top-ranking optimized basis sets as well (examples are shown in Figures S8-S15).

The third basis spectrum in Figure $3 \mathrm{~A}$ (brown line) shows the typical negative peak at $198 \mathrm{~nm}$ usually attributed to

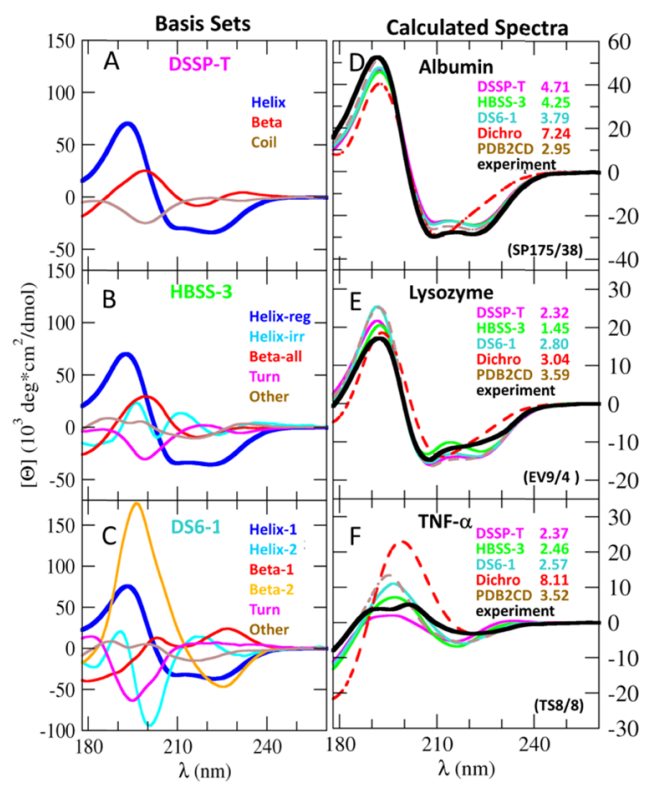

Figure 3. Comparison of basis spectrum sets and predicted CD spectra. Left column: basis spectra of three high-accuracy basis sets (DSSP-T, HBSS-3, and DS6-1). Basis spectra for similar secondary structure classes are indicated by similar colors. Right column: measured CD spectra (black solid lines) for three example proteins (human serum albumin, lysozyme, and tumor necrosis factor $\alpha$ ) compared to predicted CD spectra from three SESCA basis sets shown in the left column, DichroCalc (Dichro), and PDB2CD. The respective RMSD of the measured spectrum is given for each method in the plots in $10^{3} \mathrm{deg} \cdot \mathrm{cm}^{2} / \mathrm{dmol}$ ( $\mathrm{kMRE}$ ) units.

random coil structures. Notably, the basis sets with more detailed SS classifications in Figure 3B,C allow us to more precisely attribute this feature to certain turn types (turn-cap, $\beta$ bulge, turn type I) and extended structures (poly-proline-helical and $\beta$-cap SS elements) as represented by the turn and helix 2 classes (Figure 3C, cyan and magenta). The fact that no such peak is seen in the basis spectrum representing the remaining unassigned structures (basis sets: Other) in Figure 3 B,C shows that the negative peak previously assigned to "random coil" can indeed be mainly attributed to these three secondary structure elements. The fact that different SS subclassifications yield similar average fitting and prediction accuracies can be exploited to enhance the sensitivity for particular SS changes, e.g., from left-handed helices to parallel $\beta$-strands.

In summary, using different SS classifications, we derived several basis sets that predict the experimental protein spectra. All of these comprise three to six basis spectra, which seem to provide the best trade-off between available information content and overfitting due to experimental inaccuracies. Consistently, the measured CD spectra are predicted with an accuracy of $\sim 3.3 \mathrm{kMRE}$ ( $\mathrm{RMSD}_{\text {cross }}$ ) while avoiding overfitting.

Performance Comparison. To evaluate the performance of our method, we used the SP175 and TS8 data sets to compare SESCA with the other two available CD calculation methods outlined in the introduction, DichroCalc and PDB2CD. We emphasize that these algorithms represent different approaches of quantitative predictions based on $\mathrm{CD}$ spectroscopy, and DichroCalc, being an ab initio spectrum calculation method, was not parametrized to reproduce any particular protein reference set. In contrast, PDB2CD was developed based on the SP175 reference protein set, which enables a fair comparison to SESCA using the two data sets 


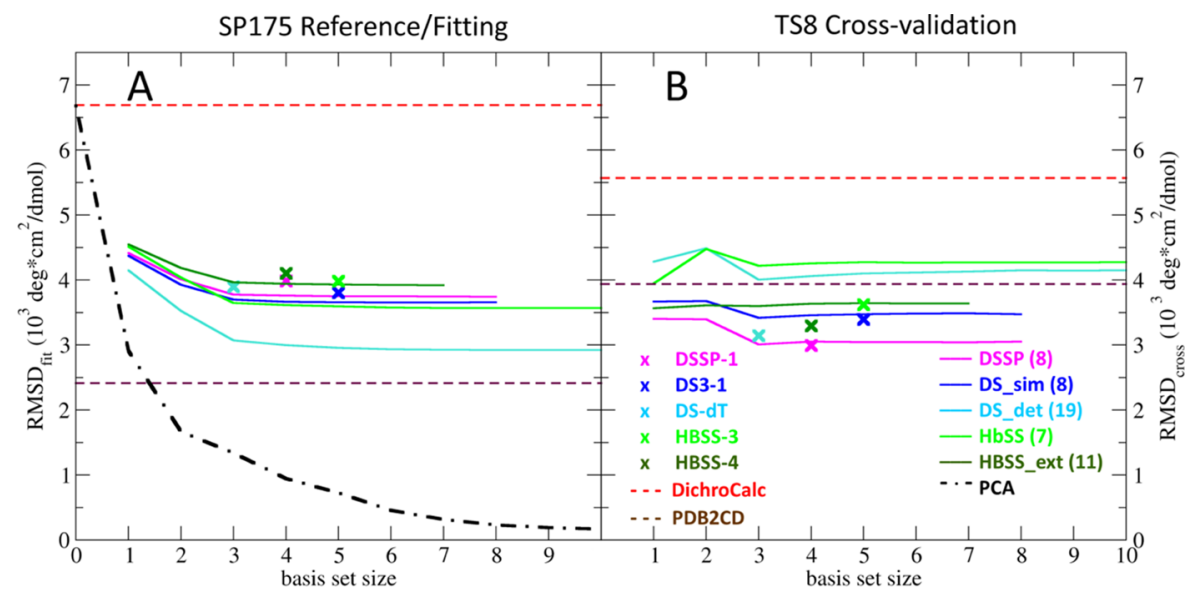

Figure 4. Fitting $\left(\mathrm{RMSD}_{\text {fit }}\right)$ and prediction $\left(\mathrm{RMSD}_{\text {cross }}\right)$ accuracies for globular proteins. Average accuracies are shown for $(\mathrm{A})$ globular proteins of the SP175 reference set, used for basis set determination and (B) the globular proteins of a small independent set TS8, used for cross-validation. The average RMSD values between measured and calculated CD spectra are shown as a function of basis set size, colored according to the SS classification protocol used (see legend on the right; the number of SS elements is shown in parentheses). Crosses mark the most predictive hard SESCA basis sets. For comparison, the maximum achievable accuracy (black dash-dotted line, determined via PCA in Section S11) is shown in (A), together with the accuracy limit of determining the CD spectra only from the SS composition of the proteins (outlined by soft SESCA basis sets derived in Section S4, shown as colored solid lines). In panel B, the accuracy of most soft basis sets (but not of hard basis sets) is reduced significantly due to overfitting. The average accuracies of DichroCalc and PDB2CD (red and brown dashed lines) are also shown on both plots to compare their predictive power with SESCA basis sets.

mentioned above. Furthermore, to avoid artifacts from the differing wavelength ranges, we restricted the RMSD calculations to the wavelength range between 178 and 260 $\mathrm{nm}$, which is accessible to all three algorithms.

Table S9 lists the average accuracies (RMSD) of the CD spectra calculated from the crystallographic structures of sets SP175 and TS8 for both DichroCalc and PDB2CD. As can be seen, the average RMSD values of CD spectra predicted by DichroCalc are $6.92 \pm 0.37$ and $5.56 \pm 1.20 \mathrm{kMRE}$ units, respectively. Interestingly, DichroCalc also predicted the CD spectra of the TS8 set at a slightly higher accuracy, suggesting that the data set may indeed have a slightly better data quality. However, these accuracies are markedly lower than those achieved by SESCA or PDB2CD. To investigate the reason for this finding, Figure $3 \mathrm{D}-\mathrm{F}$ shows $\mathrm{CD}$ spectra of three representative sample proteins: one $\alpha$-helical (D), one $\alpha / \beta$ $(\mathrm{E})$, and one $\beta$-sheet (F) protein. The comparison of the calculated CD spectra (red dashed lines) with the respective measured spectra (black lines) shows that DichroCalc only determines the most prominent spectral features, without reproducing the substructure of the peaks.

Because the purely empirical method PDB2CD calculates a weighted average of $\mathrm{CD}$ spectra measured for structurally similar reference proteins of the SP175 set, it is, unsurprisingly, markedly more accurate for this set $\left(\mathrm{RMSD}_{\mathrm{fit}}=2.40 \pm 0.26\right.$ kMRE, brown dashed lines in Figure 3D-F) than any of the SESCA basis sets, or DichroCalc. However, in contrast to DichroCalc and the optimized SESCA basis sets, its prediction accuracy for the TS8 set drops markedly $\left(\mathrm{RMSD}_{\text {cross }}=3.94 \pm\right.$ $0.60 \mathrm{kMRE}$ ), suggesting less predictive power compared to our SESCA basis sets (RMSD cross $_{\text {r }}$ ranging from 2.9 to $3.7 \mathrm{kMRE}$ ).

Note that this result differs from a previous PDB2CD crossvalidation study by Mavridis et al., ${ }^{20}$ which used a set of 14 protein structures (TS14, details are given in Table S10) and reported prediction accuracies that were very similar to their corresponding SP175 fitting accuracy. To investigate this, we performed a cross-validation on the TS14 set too and obtained an $\mathrm{RMSD}_{\text {set }}$ of $\sim 3.9 \mathrm{kMRE}$ both for SESCA basis sets and
PDB2CD, while DichroCalc performed somewhat worse $(\sim 5.6$ $\mathrm{kMRE})$. We attribute this discrepancy between the crossvalidation results to the fact that Mavridis et al. ${ }^{20}$ report deviations that are normalized by the uncertainty of the predicted spectrum, whereas we report absolute deviations. Furthermore, the TS14 set contained four $\beta$-crystallin proteins with near-identical folds, two of which were also part of the SP175 set. Since these proteins may mask overfitting during cross-validation, we removed them from the set and recalculated the average prediction accuracies for the remaining nine proteins, which yielded $\mathrm{RMSD}_{\text {set }}$ values of $4.0 \pm 0.5 \mathrm{kMRE}$ for SESCA, $4.6 \pm 0.5 \mathrm{kMRE}$ for PDB2CD, and 7.0 $\pm 1.4 \mathrm{kMRE}$ for Dichrocalc.

Because SESCA only uses the SS compositions and the precalculated basis sets, it should be computationally much more efficient than PDB2CD or DichroCalc. To test this expectation, we benchmarked all three methods using the

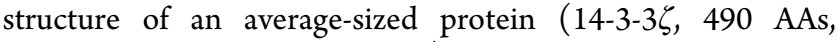
modified PDB based on 2WH0). Because the source code of PDB2CD is not available, it was benchmarked using the publicly available web server. DichroCalc and SESCA were benchmarked on a Xeon E5-1630 v4. The CD calculation with PDB2CD took 11-19 min (excluding queuing times), with most of the computation time spent on fold recognition. DichroCalc predicted the CD spectrum of $14-3-3 \zeta$ in $3-144$ min, depending on the level charge-transfer transitions and side-chain chromophores included in the calculation. In comparison, the $\mathrm{CD}$ spectrum of the same protein was calculated by SESCA in 0.3-9 s, depending on the chosen SS classification method. The high-efficiency CD predictions of SESCA are advantageous for calculating the CD spectra of large structural ensembles. It is also essential for the iterative refinement of structural ensembles, where the predicted CD spectrum has to be recalculated between refinement steps, often involving $10^{4}-10^{9}$ calculations, e.g., for MD-based refinements.

Basis Set Accuracy Limits. Next, we investigated to what extent the accuracy of our basis sets could possibly be improved (a) when including all available structural information and (b) 
when using secondary structure information only. As the best available representation of globular proteins, ${ }^{24}$ we used the SP175 reference set for this purpose. The best achievable accuracy was determined by PCA of the reference spectra as defined in Basis Set Quality Assessment (see SM Section S5 for details). By definition, the fitting accuracy $\mathrm{RMSD}_{\text {fit }}$ achieved using the first $n$ principal components provides the upper accuracy limit for a basis set of size $n$ (see the chapter on dimensionality reduction in ref 36 ).

Figure 4A shows this absolute limit (black dotted-dashed line) as a function of basis set size. For example, with two basis spectra, even when using the full structural information, an accuracy not better than $1.67 \mathrm{kMRE}$ can be achieved. This optimal accuracy rapidly decreases from an initial $6.68 \mathrm{kMRE}$ (using only the average spectrum and no structural information) to $1.34 \mathrm{kMRE}$ using up to three basis spectra, followed by a more gradual decrease to $0.23 \mathrm{kMRE}$ for eight basis spectra and a slow decline to $0.17 \mathrm{kMRE}$ at 10 basis spectra. Therefore, for accurate predictions, at least three basis spectra are required, whereas use of more than eight basis spectra does not increase the achievable accuracy further, in line with the size range of optimized basis sets obtained in Secondary Structure-Based CD Calculations.

Because the actual achievable accuracy of our basis sets is reduced by the limited structural information contained in the SS composition, as well as by the error in determining this composition from the crystal structure of the protein, we also determined the best achievable accuracy when predicting the spectra from SS information. To account for these limitations, we determined basis sets using the soft optimization method (described in Basis Set Quality Assessment), which varies the SS assignment and the shape of basis spectra iteratively to acquire the best possible fitting accuracy (details in Section S4).

The colored lines in Figure 4A show the fitting accuracy $\left(\mathrm{RMSD}_{\mathrm{fit}}\right)$ of the acquired soft basis sets for every possible basis set size using the SS information determined by the five structure classification protocols described in Secondary Structure Determination (DSSP, DS det, DS sim, HbSS, and HbSS ext). Although these lines also decrease monotonically with the basis set size, the best accuracy of only $3.5 \pm 0.6 \mathrm{kMRE}$ is achieved, depending on the SS classification, with the lowest $\mathrm{RMSD}_{\text {fit }}$ of $2.9 \mathrm{kMRE}$ obtained for the DS det protocol with 19 SS elements (light blue line). The $\sim 2.7$ kMRE difference between PCA and DS_det basis sets shows that the limited information of the SS composition (derived from a crystal structure) markedly reduces the accuracy of the predicted CD spectra.

The fitting accuracies achieved by the most predictive optimized basis sets (marked by crosses in Figure 4A, data in Table S8) are all within 0.8 kMRE of the determined accuracy limit for their respective size and SS classification. This also indicates that further significant improvements in basis set accuracies are unlikely based only on the crystal-structurederived SS information.

Figure 4B compares the predictive power of hard (crosses) and soft (lines) basis sets, as determined by cross-validation. As can be seen, the average prediction accuracy of hard basis sets $\left(\mathrm{RMSD}_{\text {cross }}=3.3 \pm 0.6 \mathrm{kMRE}\right)$ is also within the accuracy limits established above $(2.9 \pm 0.6 \mathrm{kMRE})$, which indicates that overfitting is largely avoided. In comparison, overfitting seems to be more severe for the soft basis sets; unlike their fitting accuracies in Figure 4A, prediction accuracies for soft basis sets are systematically lower than for hard basis sets and, in addition, do not improve monotonically with basis set size. The overfitting seems to be particularly severe for basis sets using SS classification protocols with more than eight structural elements (DS_det and HbSS_ext, light blue and green lines, respectively). Because of their lower predictive power, soft basis sets are not considered further for predicting protein spectra in this study.

It is instructive to also compare the average RMSD values obtained from DichroCalc and PDB2CD to the accuracy limits determined above. The calculated average RMSD values of DichroCalc (Figure 4, red dashed lines) are close to what can be achieved using only the averaged CD spectrum of the SP175 set (6.7 kMRE, PCA-0). The observed variation of the individual RMSD values is large, however, and some of the DichroCalc predictions are rather accurate (best RMSD 1.9 kMRE). On the other hand, the RMSD $\mathrm{Pit}_{\text {fit }}$ obtained by PDB2CD (2.4 kMRE) is even below that of the soft SESCA basis sets; however, a similarly good accuracy is not reached by PDB2CD during cross-validation, where hard SESCA basis sets yield higher predictive power.

Model Validation Based on CD Predictions. The CD spectrum predictions characterized above enable us to address our main question: Can one assess the quality of a protein structural model from the deviation (RMSD) between its predicted and measured CD spectra? Specifically, we focus on two questions: (1) Given a RMSD value, how large is the total error $(\Delta S S)$ in the model's secondary structure composition and (2) given two proposed models, what is the minimum difference in the $S S$ composition $\left(\Delta S S_{\text {min }}\right)$ to reliably discriminate between the two?

To answer these questions, one needs to determine how $\mathrm{RMSD}_{j}$ between the measured and the predicted spectra of protein $j$ depends on $\Delta S_{j}$, which is described in Structure Validation. In summary, to calculate $\Delta S S_{j}$, two parameters are required: $m_{j}$, which defines the proportionality between $\Delta S S$ and the SS-dependent error of the predicted CD spectrum $\left(M_{j}\right.$ $\left.=m_{j} \cdot \Delta S_{j}\right)$, and $\mathrm{RMSD}_{j}^{0}$, the average SS-independent error of the predicted spectrum, and provides an offset for $\mathrm{RMSD}_{j}$ if the SS-dependent error is small or the error terms are correlated.

Equation 5 in Structure Validation allows one to calculate $\Delta S S_{j}$ for any structural model of protein $j$ from $\mathrm{RMSD}_{j}$, provided that one knows $m_{j}, \mathrm{RMSD}_{j}^{0}$, and the error terms for protein $j$ are statistically independent. Unfortunately, both parameters depend on the correct SS composition $C_{j i}^{0}$, which is typically unknown. Furthermore, it is not known if the two error terms for a given protein are actually statistically independent. To address these problems, in Section S8, we developed an error model for each basis set that enables one to estimate $\Delta S_{j}$ without prior knowledge of $C_{j i}^{0}$ allowing for possibly correlated error terms. The model is based on fitting the average parameters $m_{\mathrm{f}}$ and $R_{\mathrm{f}}$ to the $\Delta \mathrm{SS}_{j}$ vs $\mathrm{RMSD}_{j}$ values of SP175 reference proteins, which were used to derive the basis sets. Note that, as shown in Figure S16, $\Delta S_{j}$ for each reference protein of the set was obtained by predicting its SS composition from the reference spectrum (through deconvolution, see Section S7) and comparing it to the SS composition of its reference structure. Similarly, $\mathrm{RMSD}_{j}$ values were obtained by predicting $\mathrm{CD}$ spectra from the reference structure and by comparing them to the reference spectra.

In Figure 5A, the accuracy of our error model is assessed for the DS-dT basis set. For this basis set, we obtained $m_{\mathrm{f}}=31.3 \pm$ $1.3 \mathrm{kMRE}$ and $R_{\mathrm{f}}=1.79 \pm 0.52 \mathrm{kMRE}$, which agree well with the mean of the individual $m_{j}$, and $\operatorname{RMSD}_{j}^{0}$ parameters 


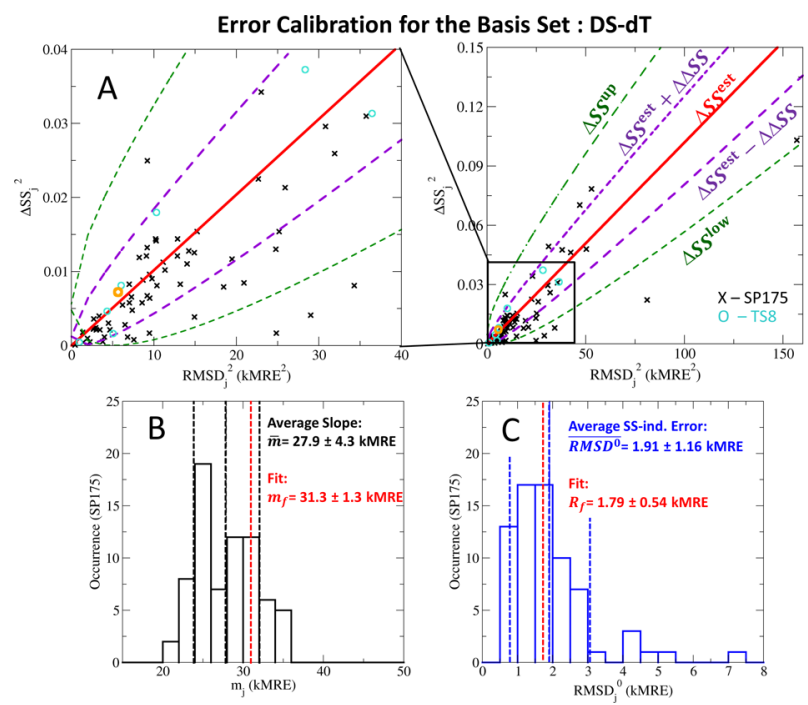

Figure 5. Error calibration for the basis set DS-dT. (A) The error in secondary structure composition $\left(\Delta S_{j}\right)$ for a proposed structural

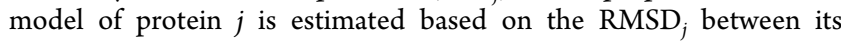
predicted and the measured CD spectra. The best estimate of $\Delta S S_{j}$ is shown as a red solid line. The estimated upper and lower bounds of $\Delta \mathrm{SS}_{j}$ are shown as dark green dashed lines. The prediction RMSD vs the real value of $\Delta S S_{j}$ for the 71 proteins in the SP175 data set are shown as black crosses. The reference proteins of the TS8 crossvalidation data set are shown as light blue circles, with a representative example, prealbumin, indicated in orange. (B) and (C) show the distribution of the SS-independent error $\left(\mathrm{RMSD}_{j}^{0)}\right.$ and the slope of the SS-dependent error $\left(m_{j}\right)$ for all proteins in the SP175 reference set, determined by deconvolution of their measured $\mathrm{CD}$ spectra. The fit parameters for our error model representing the average of these properties are shown as vertical, red dashed lines. The average error of the estimated $\Delta \mathrm{SS}_{j}( \pm \Delta \Delta \mathrm{SS}$, in purple $)$ is also indicated.

calculated from the obtained $C_{j i}^{0}$, whose distributions are shown in Figure 3B,C, respectively. Using these fit parameters, the best estimate $\Delta \mathrm{SS}_{j}^{\text {est }}=\mathrm{RMSD}_{j} / m_{\mathrm{f}}$ for a given $\mathrm{RMSD}_{j}$ (red line in Figure 5A) reproduced the $\Delta \mathrm{SS}_{j}$ values obtained with an average deviation $\left(\Delta \Delta S S_{S P 175}\right)$ of $2.6 \%$. If we allow for strong correlations between SS-dependent and SS-independent error terms, the parameter $R_{\mathrm{f}}$ and its uncertainty $\sigma_{\mathrm{R}}$ determine the uncertainty of the best estimate $\left(\sigma_{\mathrm{S}} \approx\left(R_{\mathrm{f}}+\sigma_{\mathrm{R}}\right) / m_{\mathrm{f}}\right)$. However, because the correlation between error terms is very weak for most reference proteins, a more narrow estimate for the uncertainty was also defined as $\sigma_{\mathrm{S}} \approx \Delta \Delta \mathrm{SS}_{\mathrm{SP} 175}$. The two estimated uncertainties for the DS-dT basis set are shown as green and purple dashed lines, which contained 67 (94\%) and $53(75 \%)$ of the 71 obtained reference data points within their boundaries, thereby confirming that our error estimates are indeed appropriate.

This error model now allows one to answer the first of the above two questions, to assess the quality of any given structural model by estimating $\Delta S S_{j}$ from the RMSD between its predicted and measured $C D$ spectra. As an example, we estimated $\Delta \mathrm{SS}_{j}$ for the crystal structure of prealbumin (TS8/3), a reference protein that was not used in our error model determination. The measured $\mathrm{CD}$ spectrum of prealbumin deviates by an RMSD of 2.38 kMRE from the spectrum predicted from its crystal structure using the DS-dT basis set. The error model for this basis set estimates $\Delta \mathrm{SS}_{j}$ between 0.2 and $15.5 \%$, with a $\Delta S_{j}^{\text {est }}=7.6 \%$, which is very close to the $\Delta S_{j}$
$=8.5 \%$ obtained from $\mathrm{CD}$ deconvolution (Figure 5A, orange circle).

Performing the same test on the $\Delta \mathrm{SS}_{j}$ values obtained for the TS8 cross-validation set (light blue circles in Figure 5A) showed that for the DS-dT basis set all are within the tighter estimated boundaries with an average deviation of $\Delta \Delta \mathrm{SS}_{\mathrm{TS} 8}=$ $1.7 \%$ for the best estimates. Finally, we performed the same error calibration for four optimized basis sets listed in Averaging over Prediction Results and obtained similar results with $\Delta \Delta \mathrm{SS}_{\mathrm{SP} 175}^{\text {mean }}=5.5 \%, \Delta \Delta \mathrm{SS}_{\mathrm{TS} 8}^{\text {mean }}=5.6 \%$, and with an average 95 and $75 \%$ of data points contained between the two estimated boundaries, respectively, further corroborating our approach.

To address the second question concerning the sensitivity of our model validation, we consider two proposed models with $\Delta \mathrm{SS}_{1}^{\text {est }} \pm \sigma_{\mathrm{S} 1}$ and $\Delta \mathrm{SS}_{2}^{\text {est }} \pm \sigma_{\mathrm{S} 2}$, with typical experimental errors in the reference structures and measured CD spectrum. To reliably discriminate between the two structural models, the difference $\left|\Delta S S_{2}^{\text {est }}-\Delta S S_{1}^{\text {est }}\right|$ has to be larger than

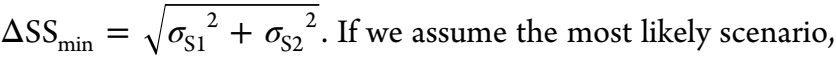
with weak correlations between the SS-dependent and SSindependent error terms for both structural models, the uncertainties $\sigma_{\mathrm{S} 1}=\sigma_{S 2}=\Delta \Delta S S_{\mathrm{SP} 175}$ and thus $\Delta \mathrm{SS}_{\min }=$ $\sqrt{ } 2 \cdot \Delta \Delta S_{\text {SP175 }}$. These assumptions yield $\Delta S_{\text {min }}$ values between $3.7 \%$ (DS-dT) and $11.2 \%$ (DS5-4) for the optimized basis sets tested. We note that both the average $\Delta S S_{j}$ and $\Delta \mathrm{SS}_{\min }$ increase with increasing basis set size. The trend in the $\Delta \mathrm{SS}_{j}$ values obtained can be explained by the larger basis sets extracting more structural information from the models, thus registering small deviations between the reference and solution structures that smaller basis sets do not detect. The increasing $\Delta \mathrm{SS}_{\min }$ values are likely due to the larger variation of $m_{j}$ and $\mathrm{RMSD}_{j}^{0}$ parameters observed for larger basis sets.

We also note that the obtained distributions of both $\mathrm{RMSD}_{j}^{0}$ (shown in Figure 5C) and $\Delta S_{j}$ values are asymmetric in the SP175 set. For over $60 \%$ of the proteins, both error terms are below the average, whereas relatively few proteins show exceptionally large errors. In particular, all four proteins outside the estimated error range depicted in Figure 5A also exhibit large SS-independent errors $\left(\mathrm{RMSD}_{j}^{0}>4 \mathrm{kMRE}\right)$, suggesting that the measured $\mathrm{CD}$ spectra of these proteins cannot be accurately predicted by the DS-dT basis set. In addition, seven proteins in the SP175 reference set have a large SS-dependent error $\left(\Delta S S_{j}>20 \%\right)$, which may have reduced the accuracy of our basis sets. To further improve the accuracy of SESCA predictions, in the next sections, we investigate several potential sources of these errors.

Improving the CD Prediction Accuracy. In Secondary Structure-Based CD calculations, we derived several SESCA basis sets to predict the $C D$ spectra of globular proteins and determined their achieved prediction accuracy. In this section, we focus on whether the prediction accuracy of our basis sets can be further improved by changing the applied methodology or the reference set. First, we study how much the use of crystal structures as structural models affects the spectrum prediction accuracy. Second, we analyze reference proteins with large CD prediction errors in our training set and their effects on the robustness of SESCA predictions. Third, we determine and include contributions from the AA side-chain groups. Finally, we present resulting improvements of the SESCA methodology through the example of a highly flexible protein complex.

Effect of the Structural Models: Solution vs Crystal

Structure. To calculate the average deviation between the SS 


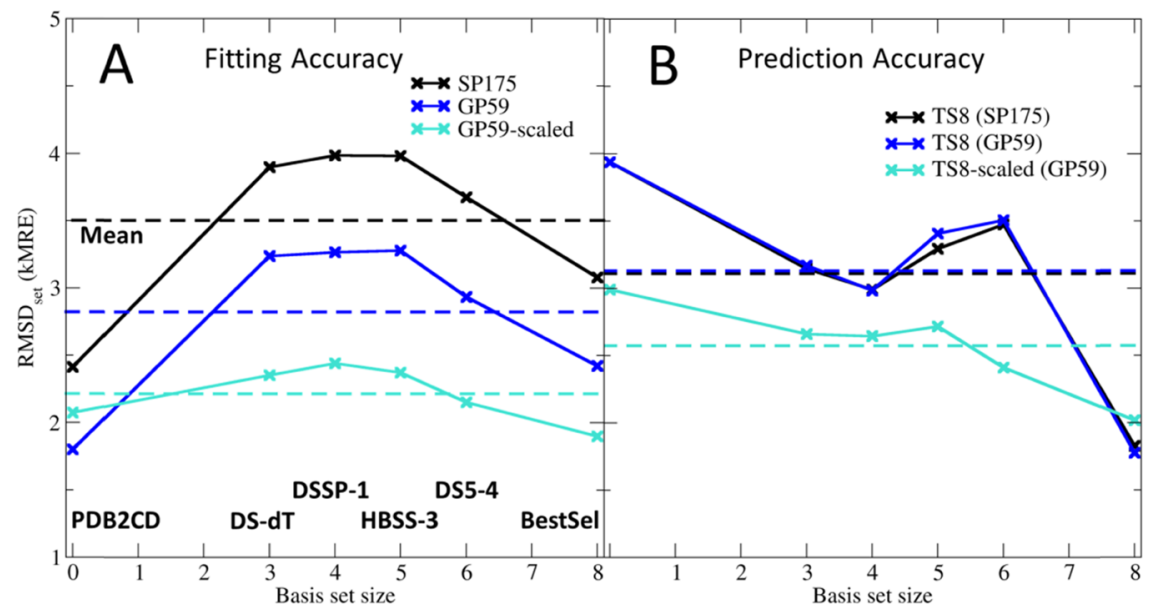

Figure 6. Changes in the mean fitting (A) and prediction accuracy (B) upon the removal of outliers and intensity scaling. Method-independent mean RMSD values (shown as dashed lines) for the fitting (SP175) and cross-validation (TS8) data sets were calculated as the average RMSD set $_{\text {of }}$ six spectrum prediction methods (crosses) including PDB2CD, four optimized SESCA basis sets of different sizes and underlying classification schemes (DS-dT, DSSP-1, HBSS-3, and DS5-4), and the Bestsel_der reconstruction basis set (see Averaging over Prediction Results). Accuracies calculated for the original unmodified data sets are shown in black, those computed after the removal of hard-to-predict proteins from the SP175 set (GP59) and subsequent recalculation of the SESCA basis spectra are shown in dark blue, and those determined after additional rescaling of measured CD intensities are shown in cyan.

composition of crystal and solution structures for globular proteins, we averaged over the total model error $\left(\Delta S S_{j}\right)$ of reference proteins determined in Basis Set Accuracy Limits. Because the individual $\Delta S_{j}$ values for the same protein scatter significantly (6.3\% on average) depending on which basis set was used to deconvolve the measured $\mathrm{CD}$ spectrum, we calculated mean deviations $\left(\Delta \mathrm{SS}_{j}^{\text {mean }}\right)$ using four optimized basis sets (see Averaging over Prediction Results). The $\Delta \mathrm{SS}_{j}^{\text {mean }}$ values show similar average differences between solution and crystal structures for the SP175 $\left(\Delta \mathrm{SS}_{\mathrm{SP} 175}^{\mathrm{mean}}=16.5 \pm 0.8 \%\right)$ and TS8 $\left(\Delta \mathrm{SS}_{\mathrm{TS} 8}^{\text {mean }}=18.0 \pm 2.3 \%\right)$ reference sets.

To assess how much these structural differences limit the accuracy of SESCA predictions, we also determined the difference between the mean prediction error $\left(\mathrm{RMSD}_{j}^{\text {mean }}\right)$ and the mean SS-independent prediction error $\left(\mathrm{RMSD}_{j}^{0, \text { mean }}\right)$ for each protein $j$, averaged over the four basis sets above. Based on these calculations, the structural differences between crystal and solution structures contribute a sizeable (up to $2.0 \mathrm{kMRE}$ ) portion of the mean fitting accuracy $\left(\mathrm{RMSD}_{\mathrm{fit}}^{\text {mean }}=3.7 \mathrm{kMRE}\right)$ for the SP175 reference set and introduce a similar error to the prediction accuracy $\left(\mathrm{RMSD}_{\text {cross }}^{\text {mean }}=3.2 \mathrm{kMRE}\right)$ for the TS8 cross-validation set.

From the above results, we conclude that use of the crystal structure is a major limitation to the accuracy of CD spectrum predictions for our reference proteins. We speculate that structural models derived from nuclear magnetic resonance (NMR) spectroscopy or MD simulations may allow more accurate $\mathrm{CD}$ spectrum predictions, as they reflect the average solution structure of the protein better. Furthermore, for several proteins of the SP175 reference set, the CD spectra were predicted with relatively poor accuracy even from the ideal SS composition, regardless of the basis set used. This points to either systematic errors in the measured CD spectra of these proteins or to strong contributions to the spectrum that cannot be predicted from the SS composition. We investigate these possibilities in the following sections.

Potential Measurement Errors of the Reference Set. Next, we asked if a particularly large mean error $\left(\mathrm{RMSD}_{j}^{\text {mean }}\right)$ of the calculated spectra points to systematic measurement errors in our reference sets. To obtain a more precise $\mathrm{RMSD}_{j}^{\text {mean }}$ for each protein in the SP175 and TS8 reference sets, we averaged over the errors $\left(\mathrm{RMSD}_{j}\right)$ of the CD spectra calculated by the four SESCA basis sets and two other methods, PDB2CD and Bestsel_der (see Averaging over Prediction Results). Then, we selected 12 proteins from the SP175 reference set (highlighted in Figure S3A and listed in S3B) and one from TS8 as particularly hard to predict because their $\mathrm{RMSD}_{j}^{\text {mean }}$ exceeded the average prediction error $\left(\mathrm{RMSD}_{\text {cross }}^{\text {mean }}\right)$ of the TS8 set (solid line) by more than one standard deviation (top dashed line) as described in Section S9. Recalculating the basis spectra of SESCA basis sets from the SP175 set without the 12 outliers (GP59 set) indeed improved the mean RMSD from 3.3 to 2.7 kMRE units (Figure 6A, black and blue lines), whereas the mean prediction accuracy of the basis sets shown in Figure $6 \mathrm{~B}$ was essentially unchanged. Therefore, we conclude that the prediction accuracy of our basis sets is robust with respect to the presence of the hard-to-predict proteins.

Interestingly, for five of the 13 outliers, the calculated CD spectra agree well with the measured CD spectra after a simple rescaling (blue in Figure S3B, example in Figure S3C). This finding suggests that at least in these cases, inaccurate intensity normalization is the major source of RMSD between calculated and measured spectra, most likely due to uncertainties in the independently measured protein concentrations. For two outliers (magenta in Figure S3B), differences in relative peak positions and intensities suggest that the large RMSD values are likely due to a combination of inaccurate intensity normalization and differences between the solution and reference structures. For the remaining six proteins (marked red, including Jacalin shown in Figure S3D), scaling factors reduced the RMSD between the measured and predicted spectra but did not yield a good agreement between the two even after fitting the SS composition of the protein model using deconvolution, which suggests additional contributions to measured CD spectra (discussed in SM Section S9).

To test whether inaccuracies in the measured concentrations may generally limit the accuracy of our CD spectrum calculations, we applied scaling factors $\left(\mathrm{SF}_{j}^{\text {mean }}\right)$ to the 

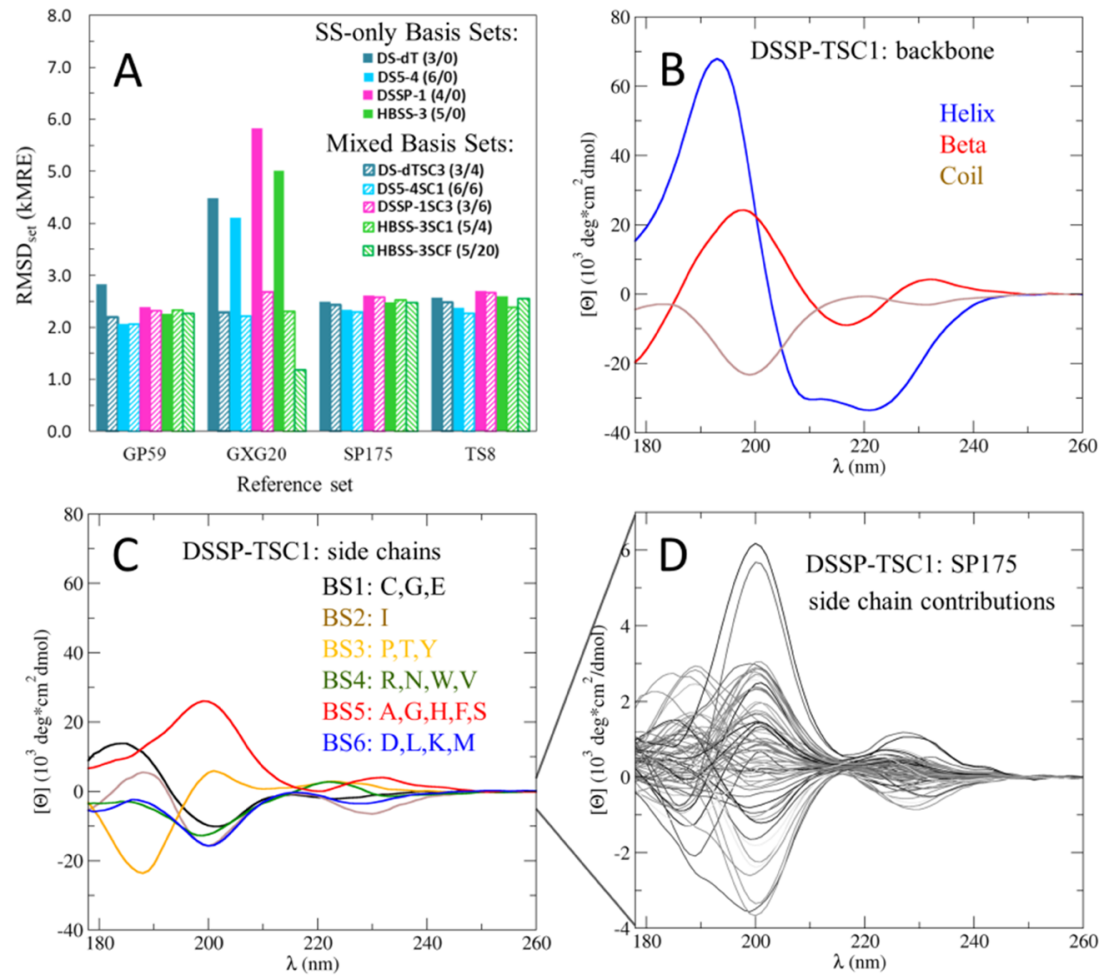

Figure 7. Comparison of backbone and side-chain contributions to the calculated CD spectra. (A) Comparison of achieved average accuracies $\left(\right.$ RMSD $_{\text {set }}$ ) for the globular protein (GP59) and short peptide (GXG20) subsets of the used training set, as well as for the SP175 reference set, and the TS8 cross-validation set. Different colors indicate different basis sets with (shaded) and without (filled) side-chain corrections; legends show the name of the basis set followed by the number of used backbone and side-chain basis spectra in parentheses. (B) Backbone and (C) side-chain basis spectra for the DSSP-TSC1 basis set; the grouping of side chains is indicated by one-letter codes of their respective AAs. (D) Combined side-chain contributions for all SP175 proteins from the DSSP-dT1SC basis set; note the magnified $y$-axis.

measured CD spectra of all proteins in the SP175 and TS8 data sets. These scaling factors were determined based on the six predicted spectra per protein from which $\mathrm{RMSD}_{j}^{\text {mean }}$ values were calculated (see Averaging over Prediction Results). First, we computed the six individual scaling factors $\left(\mathrm{SF}_{j}\right)$ that minimize the RMSD between the measured CD spectrum and one of the calculated spectra, and then we averaged them to obtain a mean scaling factor $\left(\mathrm{SF}_{j}^{\text {mean }}\right)$.

Indeed, as shown in Figure 6 (cyan lines), rescaling the measured spectrum intensities by $\mathrm{SF}_{j}^{\text {mean }}$ improved both $\mathrm{RMSD}_{\mathrm{GP} 59}^{\text {mean }}$ from 2.7 to $2.2 \mathrm{kMRE}$ and $\mathrm{RMSD}_{\mathrm{TS} 8}^{\text {mean }}$ from 3.4 to 2.7 kMRE units. The scaling factors that achieved this improvement averaged around 0.89 and 1.02 for the SP175 and TS8 reference sets, respectively, with scattering by standard deviations of 0.29 and 0.25 around these averages. The latter variations are in line with typical uncertainties of $30-15 \%{ }^{37-39}$ for the two mainly employed concentration measurement methods, quantitative amino acid analysis and UV absorption. In addition, for seven proteins in the SP175 set, independent $\mathrm{CD}$ measurements are reported by Perczel et al. ${ }^{10}$ For these proteins, the intensity of the measured $C D$ spectra differs by factors between 1.1 and 0.8 , further supporting the use of scaling factors to match our predictions.

Including Side-Chain Contributions to the CD Spectrum. Because the best achievable prediction accuracy (Performance Comparison) is still markedly higher than the ones we obtained based solely on the SS composition (even after rescaling the measured $\mathrm{CD}$ spectra), including additional information should further improve the $C D$ prediction accuracy. As the second most common type of chromophores in proteins, we therefore quantified the contribution of AA sidechain groups to protein $\mathrm{CD}$ spectra in the far-UV range and included these contributions within the SESCA scheme. We note that side-chain contributions are also considered as optional corrections in DichroCalc, and some deconvolution basis sets also include side-chain basis spectra. ${ }^{5}$

To this end, we assembled a new reference set (MP79) composed of 59 globular proteins (GP59) and 20 short peptides (GXG20) containing only a single side chain (see Protein Reference Sets for Calibration). Then, we calculated and subtracted the backbone contributions (see Section S3) and derived the average CD signal of all 20 AA side chains (shown and discussed in Section S9). Overall, we found that almost all side chains contribute markedly to the CD signal in the far-UV range (Figure S4B) and to a similar extent as the protein backbone. Notably, the obtained side-chain basis spectra also differ significantly from $C D$ spectra of individual AAs (Figure S4A).

The analysis of the PCA basis sets (Section S11) suggests that only a few basis spectra are actually required to represent the contribution of the side chains. Therefore, to obtain basis sets that can optimally represent side-chain contributions, we combined the $20 \mathrm{AA}$ side chains into classes (e.g., in Figure 7C), thus allowing each basis spectrum to represent multiple side chains. The assignment of the AA side chains to these classes was optimized by the hard optimization scheme, similarly to the assignment of SS elements for backbone contributions. Incorporating the side chains into our basis set determination protocol (described in Section S3) yielded "mixed" basis sets where some of the basis spectra represented 
A

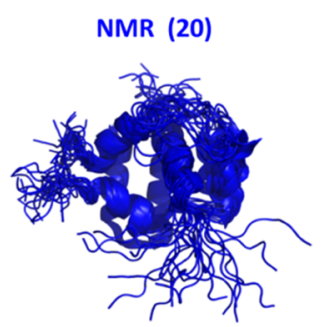

AH: $47.2 \%$ BS: $3.8 \%$ RC: $49.0 \%$
P53/CBP Complex Structural Models

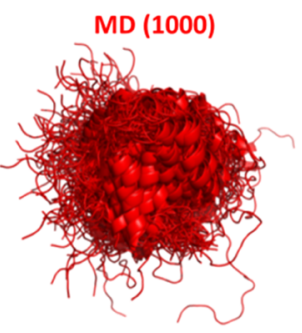

AH: $38.4 \%$ BS: $3.1 \%$ RC: $58.5 \%$

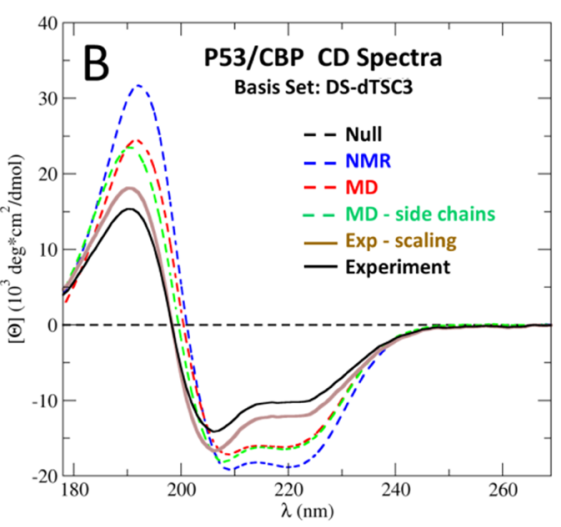

$\lambda(\mathrm{nm})$

C Improvements for CD Predicitions (DS-dTSC3)

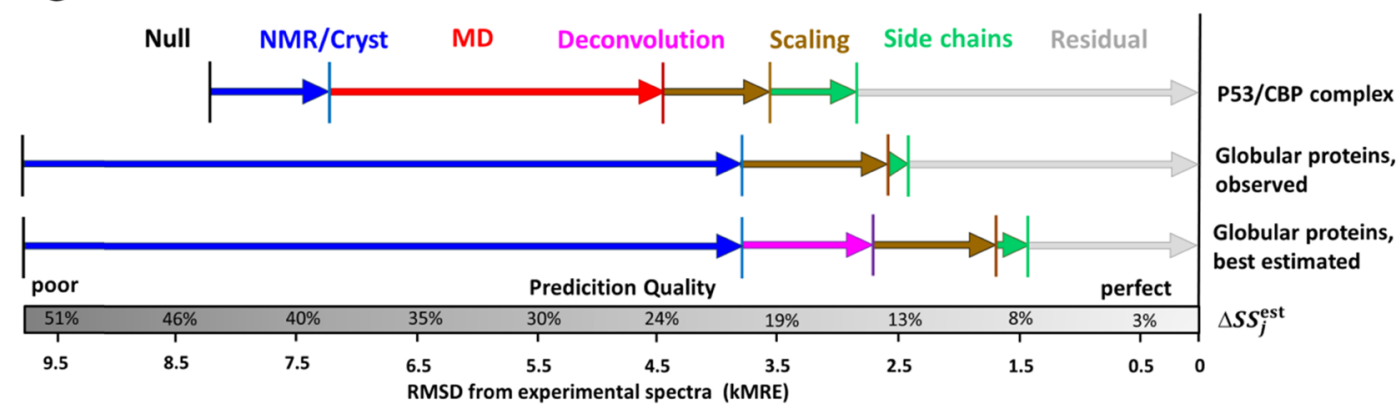

Figure 8. CD spectrum predictions and accuracy improvements for the P53/CBP protein complex. (A) Comparison of two structural ensemble models, 20 structures from a nuclear magnetic resonance bundle (NMR) and an ensemble of 1000 snapshots ('MD') extracted from a $10 \mu \mathrm{s}$ MD simulation of the same complex. The percentages below indicate average secondary structure contents, divided into $\alpha$-helix (AH), $\beta$-strand (BS), and random coil (RC). (B) Improvement of predicted CD spectra (dashed lines) with respect to measured spectra (solid lines). Predicted spectra are shown as "null" (black, $S^{\text {calc }}(\lambda)=0$ ), NMR (blue, using the NMR bundle without any further corrections), MD (red, using the MD ensemble instead), and MD-side chains (green, MD with side-chain corrections), all calculated with the SESCA basis set DS-dTSC3. The experimental CD spectrum is shown with (brown) and without (black) intensity scaling. (C) The sequence of arrows shows, to scale, the increase in prediction accuracy due to the above four improvement steps for the P53/CBP complex (top) and for the SP175 reference set (averaged, mid). The bottom arrows indicate the best achievable accuracies for the SP175 set using "perfect" secondary structure compositions obtained by CD spectrum deconvolution (magenta). The bottom gray bar translates the achieved RMSD values from the measured spectra into estimated secondary structure errors for the used models.

the SS-dependent backbone contribution of the peptide bonds, while others provided the side-chain contributions, which depend only on the AA composition.

The resulting optimized mixed basis sets (examples are shown in Figures S17-S22) typically include 3-6 backbone basis spectra and 4-7 side-chain basis spectra, with one or two basis spectra representing the positive $\mathrm{CD}$ signals of the aromatic residues. Figure $7 \mathrm{~A}$ compares the average $\mathrm{RMSD}_{\text {set }}$ 's achieved by optimized basis sets with (shaded bars) and without (solid bars) side-chain contributions. For a fair comparison, predictions for all globular proteins were compared to their rescaled reference spectra, and the basis spectra of SS-only basis sets were recalculated using the rescaled GP59 set (as discussed in Potential Measurement Errors of the Reference Set). As the figure shows, including side-chain information improves both $\mathrm{RMSD}_{\mathrm{GP59}}^{\text {mean }}$ (training) by 0.16 kMRE and RMSD TS8 $^{\text {mean }}$ (cross-validation) by $0.11 \mathrm{kMRE}$. Notably, the gain in prediction accuracy is larger (0.29 kMRE) when the mixed basis sets are compared to the original optimized basis sets while predicting the unmodified TS8 spectra. The relatively small influence of the side groups for globular proteins is in line with the PCA analysis of the SP175 spectra, which suggested an upper limit of $\sim 0.4 \mathrm{kMRE}$, and underscores the robustness of the secondary-structure-based SESCA predictions.
Given that the intensities of the backbone and the side-chain basis spectra are rather similar (Figure 7B,C shows examples for the DSSP-T1SC basis set), the improvement due to inclusion of the side-chain spectra is small. We attribute this unexpected result mainly to 2 factors. The first is the partial cancellation of contributions from side-chain basis spectra with opposite signs and similar coefficients. This is observed for most reference proteins because most globular proteins have similar AA compositions. For example, Figure $7 \mathrm{D}$ shows the total contribution of the side chains for each protein in the SP175 reference set, which, overall, are an order of magnitude smaller than the individual side-chain basis spectra. The second factor is the correlation between AA and SS compositions (Pearson coefficients between 0.2 and 0.6 for SP175), which implies that a substantial fraction of the side-chain contributions is described already by the backbone basis spectra.

A potential third factor is that the side-chain contributions strongly depend on their environment, which therefore cannot be accurately described by just one basis spectrum. Examples are contributions from buried versus solvent-accessible side chains or side chains in different protonation states. However, we will not explore this possibility further here.

Whereas including the side-chain corrections improves the predicted CD spectra only slightly for most globular proteins, the $\mathrm{RMSD}_{\text {set }}$ calculated for the GXG20 peptides decreases 
markedly by 1.9-3.2 kMRE because their CD spectra are largely defined by the side-chain signals. This result suggests that the side-chain contributions might be more important for the prediction of CD spectra of small peptides or proteins with unusual AA compositions such as the low-complexity regions and sequence repeats often found in intrinsically disordered proteins. Therefore, in the next section, we analyze a system composed of two disordered proteins to test if side chains indeed play a larger role in predicting their CD spectra.

Case Study: CD Predictions for a Flexible Protein Complex. To quantify the outlined improvements in prediction accuracy due to model quality, spectrum rescaling, and side-chain contributions, we selected the flexible protein complex formed by the two IDP domains P53-AD2 and CBP$\mathrm{NCBD}^{40}$ as a test system. These domains form an ordered complex, the structure of which (PDB code 2L14) was determined by NMR spectroscopy. We chose this complex because without the above improvements, a poor mean prediction accuracy (an $\mathrm{RMSD}_{j}^{\text {mean }}=5.07 \mathrm{kMRE}$ and $\sigma_{j}=$ $1.58 \mathrm{kMRE}$ ) was achieved by the six methods described in Averaging over Prediction Results, and only the HBSS-3 basis set predicted the $C D$ spectrum with above average accuracy (2.0 kMRE). In this sense, it is a particularly challenging test system.

To put the prediction accuracy improvements in perspective, we first added a "null" prediction accuracy as the RMSD between the measured spectrum (Figure 8B, black solid line) and a "flat line prediction" $S_{j l}^{\text {calc }}=0($ RMSD $=8.23 \mathrm{kMRE}$, black dashed line). Then, we predicted the CD spectrum of the P53/ CBP complex from the unmodified NMR bundle shown (Figure 8A left, blue), which contained 20 conformations. For the CD predictions, we used the mixed basis set DS-dT3SC, as it has both high accuracy ( RMSD $_{\text {cross }}=3.72 \mathrm{kMRE}$ ) and sensitivity $\left(\Delta S S_{\min }=6.9 \%\right)$. The first predicted spectrum (Figure $8 \mathrm{~B}$, blue dashed line) was calculated with only the backbone basis spectra without scaling or side-chain corrections and differed from the measured spectrum by an RMSD of 7.23 kMRE (Figure 8C, "NMR/Cryst").

To probe the effects of the underlying structural model on prediction accuracy, we generated a second model for the P53/ CBP complex (Figure 8A right, red). This model was a structural ensemble of 1000 conformations obtained from an MD simulation, which was started from the NMR model as described in Structural Models and Molecular Dynamics Simulations. The MD ensemble described conformational heterogeneity and flexibility of the system, and its SS composition contained $9.5 \%$ more random coil than the NMR bundle. The MD model was validated by predicting the average backbone NMR chemical shifts from both models using the Sparta+ program ${ }^{41}$ and by comparing the resulting chemical shift profiles (discussed in Section S12) with the original NMR measurements. The lower average deviation of the calculated $\mathrm{C}_{\alpha}$ secondary chemical shifts (1.06 vs $1.39 \mathrm{ppm}$ ), which are strongly correlated with the SS, suggests that the MD model represents the SS composition of the P53/CBP complex in solution better than the original NMR model.

As expected, the RMSD of the spectrum predicted from the MD model (Figure $8 \mathrm{~B}$, red) is markedly smaller (4.45 kMRE), improving the prediction accuracy by $2.48 \mathrm{kMRE}$ (Figure $8 \mathrm{C}$, red arrow). Additionally, the better model also improved the $\mathrm{RMSD}_{j}^{\text {mean }}$ of the complex by $2.0 \mathrm{kMRE}$ when the CD spectra were predicted using the four SESCA basis sets listed in Averaging over Prediction Results.
Next, we assessed the effect of intensity scaling. To this end, we determined a mean scaling factor $\left(\mathrm{SF}_{j}^{\text {mean }}=1.18\right)$ by averaging over the optimal scaling factors required to match the spectra predicted from the MD model using the four basis sets mentioned above. For the DS-dTSC3 basis set, rescaling the measured CD spectrum by 1.18 (Figure $8 \mathrm{~B}$, brown) further reduced the prediction RMSD for the MD model from 4.45 to 3.59 kMRE (Figure 8C, brown arrow).

Finally, including side-chain contributions shifts the peaks of the predicted spectrum (Figure $8 \mathrm{~B}$, green) between 190 and $210 \mathrm{~nm}$ and reduces the deviation from the rescaled spectrum to $\mathrm{RMSD}_{j}=2.85 \mathrm{kMRE}$ (Figure $8 \mathrm{C}$, green arrow). Altogether, the applied corrections improve the prediction accuracy for the P53/CBP complex by $4.1 \mathrm{kMRE}$ (7.23 vs $2.85 \mathrm{kMRE),}$ indicating that such corrections may allow accurate SESCA predictions for other IDPs as well.

For comparison, the CD spectrum of the P53/CBP complex was also predicted from the NMR model by DichroCalc (6.16 kMRE, without side chains) and PDB2CD (7.79 kMRE). Unfortunately, for technical reasons, predicting the CD spectrum from the MD model was not possible using these methods. Rescaling the measured spectrum improves the RMSD for both methods to 5.03 and 6.50 kMRE, respectively. Additional accounting for side-chain contributions improves the RMSD further for DichroCalc to a remarkable $3.46 \mathrm{kMRE}$. Thus, when both scaling and side-chain corrections are applied, the prediction RMSD of DichroCalc is between 2.85 and 5.62 kMRE, the values obtained using the tested SESCA basis set with and without accounting for the conformational flexibility by $\mathrm{MD}$.

The second series of arrows in Figure 8C (globular proteins, observed) shows the corresponding average RMSD improvements achieved for the SP175 reference set using the same DSdTSC3 basis set. We followed the same steps as outlined for the P53/CBP complex, except that improvements due to MD simulations were not calculated, as validated MD simulations were mostly not available. As indicated by the gray bar at the bottom of Figure $8 \mathrm{C}$, the average quality of the (mostly) crystal structures in the reference set $\left(\mathrm{RMSD}_{\text {set }}=3.73 \mathrm{kMRE}, \Delta \mathrm{SS}_{\text {set }}^{\text {est }}=\right.$ $20 \%)$ is similar to that obtained for the P53/CBP complex by NMR spectroscopy with subsequent MD simulation $\left(\Delta S_{j}^{\text {est }}=\right.$ $24 \%$ ). Using these structural models, $\mathrm{RMSD}_{\text {set }}$ improves mostly due to intensity scaling (1.21 kMRE, brown arrow), while sidechain corrections, as expected, yield much smaller average improvements (0.16 kMRE, green arrow) compared to the IDP complex (0.74 kMRE). Applying the above corrections also reduces the estimated model errors to $13 \%$ for the SP175 set and to $15 \%$ for the P53/CBP complex, respectively.

Finally, we also calculated an upper limit for the prediction accuracy that can be achieved with "perfect" structure models, obtained by deconvolving (see Section S7) the rescaled and side-chain corrected CD spectra with the backbone basis spectra. Applying all further improvements, as outlined above, results in the third sequence of arrows (Figure $8 \mathrm{C}$, globular proteins, best estimated), and an optimal $\mathrm{RMSD}_{\text {set }}=1.43$ kMRE is achieved.

\section{CONCLUSIONS}

In this study, we presented a new structure-based empirical spectrum calculation approach (SESCA) to predict the electronic circular dichroism (CD) spectra of proteins from their secondary structure (SS) composition, which in turn was derived from their model structures. For several structure 
classification algorithms (DSSP, DISICL, and HbSS), basis spectrum sets were derived and their prediction accuracies were assessed by comparison to measured CD spectra.

All basis spectra were derived and optimized using a reference set consisting of 71 globular proteins; subsequently, the prediction accuracies of the basis sets were determined by cross-validation on a second, non-overlapping set of eight selected proteins, covering a broad range of SS contents. SESCA predicts the experimental CD spectra of these proteins with an average root-mean-squared deviation (RMSD) as small as $3.3 \pm 0.6 \times 10^{3} \mathrm{deg} \cdot \mathrm{cm}^{2} / \mathrm{dmol}(3.3 \pm 0.6 \mathrm{kMRE})$ in mean residue ellipticity units or $0.9 \pm 0.2 \mathrm{M}^{-1} \mathrm{~cm}^{-1}$ in $\Delta \varepsilon$ units. This prediction accuracy is markedly better than that of the best currently available algorithms PDB2CD (3.9 kMRE) and DichroCalc (5.6 kMRE).

Closer analysis of the optimized basis sets shows that the best possible prediction accuracy given the current reference set and SS information was reached using 3-8 basis spectra and that this prediction accuracy is similar for all of the tested SS classification protocols.

Further, we demonstrate that SESCA enables one to validate structural models based on the RMSD between its predictions and measured protein CD spectra. Depending on the basis set used, our results show that SESCA is able to discriminate between structural models differing as little as $4-12 \%$ in their SS composition. Using this methodology and the measured CD spectra, we estimate that, on average, the SS composition of model structures in our reference sets differs by $15-20 \%$ from the solution structure of their respective proteins.

Investigating 13 reference proteins of which the CD spectra were particularly hard to predict, we determined that these SS differences, together with inaccurate normalization of the measured CD spectra, are the major factors limiting our prediction accuracy. Nevertheless, even though inaccurate protein models and CD spectra lead to poor RMSD values for some of the reference proteins, our CD spectrum prediction method proved to be relatively robust with respect to these outliers in the training set.

Rescaling the reference spectra, discarding the worst outliers from the training set, and accounting for AA side-chain contributions via an additional 4-7 basis spectra, we derived "mixed" basis sets that improved the prediction accuracy of SESCA to $2.5 \pm 0.3 \mathrm{kMRE}$.

For globular proteins, including side-chain contributions yielded only small (on average, less than $0.2 \mathrm{kMRE}$ ) improvements for $\mathrm{CD}$ predictions. In contrast, for small peptides as well as for a sample complex of two intrinsically disordered protein (IDP) domains, namely, P53-AD2 and CBP-NCBD (P53/CBP), side-chain contributions improved the prediction accuracies substantially.

Due to the simple SS calculations involved, as well as the use of precalculated basis sets, SESCA is computationally highly efficient and can be applied to rather large structural ensembles. This feature allows one to go beyond average structures and to account for effects of a protein's conformational flexibility on its CD spectrum. Indeed, for the P53/CBP complex, an extended molecular dynamics trajectory improves the accuracy of the calculated CD spectrum considerably (by more than 2.0 kMRE).

Specifically, the example of the P53/CBP complex suggests that SESCA may be particularly helpful in modeling IDPs. These biologically highly relevant molecules are notoriously hard to characterize, and structural ensembles are usually required to understand their conformational flexibility, which is often closely related to the IDP function. A more systematic assessment of SESCA performance regarding IDPs will be addressed in a separate study.

Here, we have exploited the high sensitivity of $C D$ spectroscopy to the average SS of $\alpha$-L-amino acid polypeptides to evaluate and improve protein structural models. Our SESCA is not restricted to proteins and peptides, however. Because CD spectroscopy is also sensitive to the structures of polynucleic acids (DNA and RNA), modified polypeptides (e.g., by posttranslational modifications or ones composed of unnatural amino acids), and certain carbohydrates (such as glycan structures), SESCA can also be applied to these biomolecules as soon as appropriate reference data sets become available.

\section{ASSOCIATED CONTENT}

\section{Supporting Information}

The Supporting Information is available free of charge on the ACS Publications website at DOI: 10.1021/acs.jctc.9b00203.

Additional information about the derived basis sets and their performance, technical details about the basis set derivation and optimization process, as well as detailed analysis of potential experimental errors in the reference structures and spectra; a python implementation of our CD calculation method SESCA, basis sets, and tools compatible with the SS classification algorithms DISICL, DSSP, and HBSS are publicly available online: http:// www.mpibpc.mpg.de/sesca (PDF)

\section{AUTHOR INFORMATION}

\section{Corresponding Author}

*E-mail: hgrubmu@gwdg.de. ORCID $\odot$

Gabor Nagy: 0000-0002-8607-9682

Maxim Igaev: 0000-0001-8781-1604

Nykola C. Jones: 0000-0002-4081-6405

Søren V. Hoffmann: 0000-0002-8018-5433

Helmut Grubmüller: 0000-0002-3270-3144

Notes

The authors declare no competing financial interest.

\section{ACKNOWLEDGMENTS}

The authors would like to thank J. Kardos and A. Micsonai for providing $C D$ spectra and secondary structure data of the SP175 protein data set used for training the BeStSel algorithm; to J. Hritz, S. Becker, and C. Griesinger for providing protein samples for the CD measurements; B. Gyurcsik for the aid with the experimental design; and P. Kellers for editing the manuscript. We would like to thank the storage ring facilities of Aarhus University (ISA) for awarding beam time on the AUCD beam line, ASTRID2, for the measurements of the CD spectra of the short peptides. This research project was funded and supported by the Alexander von Humboldt Foundation and the Max Planck Society.

\section{REFERENCES}

(1) Circular Dichroism and the Conformational Analysis of Biomolecules; Fasman, G. D., Ed.; Springer US: Boston, MA, 1996.

(2) Brahms, S.; Brahms, J. Determination of Protein Secondary Structure in Solution by Vacuum Ultraviolet Circular Dichroism. J. Mol. Biol. 1980, 138, 147-178. 
(3) Kelly, S. M.; Jess, T. J.; Price, N. C. How to Study Proteins by Circular Dichroism. Biochim. Biophys. Acta, Proteins Proteomics 2005, 1751, 119-139.

(4) Johnson, W. C., Jr. Protein Secondary Structure and Circular Dichroism: A Practical Guide. Proteins 1990, 7, 205-214.

(5) Hennessey, J. P., Jr.; Johnson, W. C., Jr. Information Content in the Circular Dichroism of Proteins. Biochemistry 1981, 20, 1085-1094.

(6) Goodman, M.; Toniolo, C. Conformational Studies of Proteins with Aromatic Side-Chain Effects. Biopolymers 1968, 6, 1673-1689.

(7) Strickland, E. H.; Beychok, S. Aromatic Contributions To Circular Dichroism Spectra Of Protein. Crit. Rev. Biochem. 1974, 2, 113-175.

(8) Chakrabartty, A.; Kortemme, T.; Padmanabhan, S.; Baldwin, R. L. Aromatic Side-Chain Contribution to Far-Ultraviolet Circular Dichroism of Helical Peptides and Its Effect on Measurement of Helix Propensities. Biochemistry 1993, 32, 5560-5565.

(9) Micsonai, A.; Wien, F.; Kernya, L.; Lee, Y.-H.; Goto, Y.; Réfrégiers, M.; Kardos, J. Accurate Secondary Structure Prediction and Fold Recognition for Circular Dichroism Spectroscopy. Proc. Natl. Acad. Sci. USA 2015, 112, E3095-E3103.

(10) Perczel, A.; Hollósi, M.; Tudnady, G.; Fasman, G. D. Convex Constraint Analysis: A Natural Deconvolution of Circular Dichroism Curves of Proteins. Protein Eng., Des. Sel. 1991, 4, 669-679.

(11) Louis-Jeune, C.; Andrade-Navarro, M. A.; Perez-Iratxeta, C. Prediction of Protein Secondary Structure from Circular Dichroism Using Theoretically Derived Spectra. Proteins: Struct., Funct., Bioinf. 2012, 80, 374-381.

(12) Sreerama, N.; Woody, R. W. Estimation of Protein Secondary Structure from Circular Dichroism Spectra: Comparison of CONTIN, SELCON, and CDSSTR Methods with an Expanded Reference Set. Anal. Biochem. 2000, 287, 252-260.

(13) Greenfield, N. J. Methods to Estimate the Conformation of Proteins and Polypeptides from Circular Dichroism Data. Anal. Biochem. 1996, 235, 1-10.

(14) Johnson, W. C. Analyzing Protein Circular Dichroism Spectra for Accurate Secondary Structures. Proteins: Struct., Funct., Bioinf. 1999, 35, 307-312.

(15) Štěpánek, P.; Bouř, P. Multi-Scale Modeling of Electronic Spectra of Three Aromatic Amino Acids: Importance of Conformational Averaging and Explicit Solute-solvent Interactions. Phys. Chem. Chem. Phys. 2014, 16, 20639-20649.

(16) Fukuyama, T.; Matsuo, K.; Gekko, K. Vacuum-Ultraviolet Electronic Circular Dichroism of L-Alanine in Aqueous Solution Investigated by Time-Dependent Density Functional Theory. J. Phys. Chem. A 2005, 109, 6928-6933.

(17) Oakley, M. T.; Bulheller, B. M.; Hirst, J. D. First-Principles Calculations of Protein Circular Dichroism in the Far-Ultraviolet and Beyond. Chirality 2006, 18, 340-347.

(18) Bayley, P. M.; Nielsen, E. B.; Schellman, J. A. The Rotatory Properties of Molecules Containing Two Peptide Groups: Theory. J. Phys. Chem. A 1969, 73, 228-243.

(19) Bulheller, B. M.; Hirst, J. D. DichroCalc-Circular and Linear Dichroism Online. Bioinformatics 2009, 25, 539-540.

(20) Mavridis, L.; Janes, R. W. PDB2CD: A Web-Based Application for the Generation of Circular Dichroism Spectra from Protein Atomic Coordinates. Bioinformatics 2017, 33, 56-63.

(21) Sreerama, N.; Venyaminov, S. Y.; Woody, R. W. Estimation of the Number of A-Helical and B-Strand Segments in Proteins Using Circular Dichroism Spectroscopy. Protein Sci. 1999, 8, 370-380.

(22) Mao, D.; Wachter, E.; Wallace, B. A. Folding of the Mitochondrial Proton Adenosine Triphosphatase Proteolipid Channel in Phospholipid Vesicles. Biochemistry 1982, 21, 4960-4968.

(23) Berman, H. M.; Westbrook, J.; Feng, Z.; Gilliland, G.; Bhat, T. N.; Weissig, H.; Shindyalov, I. N.; Bourne, P. E. The Protein Data Bank. Nucl. Acids Res. 2000, 28, 235-242.

(24) Lees, J. G.; Miles, A. J.; Wien, F.; Wallace, B. A. A Reference Database for Circular Dichroism Spectroscopy Covering Fold and Secondary Structure Space. Bioinformatics 2006, 22, 1955-1962.
(25) Kabsch, W.; Sander, C. Dictionary of Protein Secondary Structure: Pattern Recognition of Hydrogen-Bonded and Geometrical Features. Biopolymers 1983, 22, 2577-2637.

(26) Orengo, C. A.; Michie, A. D.; Jones, S.; Jones, D. T.; Swindells, M. B.; Thornton, J. M. CATH-a Hierarchic Classification of Protein Domain Structures. Structure 1997, 5, 1093-1109.

(27) Whitmore, L.; Woollett, B.; Miles, A. J.; Klose, D. P.; Janes, R. W.; Wallace, B. A. PCDDB: The Protein Circular Dichroism Data Bank, a Repository for Circular Dichroism Spectral and Metadata. Nucleic Acids Res. 2011, 39, D480-D486.

(28) Kuipers, B. J. H.; Gruppen, H. Prediction of Molar Extinction Coefficients of Proteins and Peptides Using UV Absorption of the Constituent Amino Acids at $214 \mathrm{Nm}$ To Enable Quantitative Reverse Phase High-Performance Liquid Chromatography-Mass Spectrometry Analysis. J. Agric. Food Chem. 2007, 55, 5445-5451.

(29) Abraham, M. J.; Murtola, T.; Schulz, R.; Páll, S.; Smith, J. C.; Hess, B.; Lindahl, E. GROMACS: High Performance Molecular Simulations through Multi-Level Parallelism from Laptops to Supercomputers. SoftwareX 2015, 1-2, 19-25.

(30) Huang, J.; Rauscher, S.; Nawrocki, G.; Ran, T.; Feig, M.; de Groot, B. L.; Grubmüller, H.; MacKerell, A. D. CHARMM36m: An Improved Force Field for Folded and Intrinsically Disordered Proteins. Nat. Methods 2017, 14, 71-73.

(31) MacKerell, A. D., Jr; Bashford, D.; Bellott, M.; Dunbrack Jr, R. L.; Evanseck, J. D.; Field, M. J.; Fischer, S.; Gao, J.; Guo, H.; Ha, S.; et al. All-Atom Empirical Potential for Molecular Modeling and Dynamics Studies of Proteins. J. Phys. Chem. B 1998, 102, 3586-3616.

(32) Nagy, G.; Oostenbrink, C. Dihedral-Based Segment Identification and Classification of Biopolymers I: Proteins. J. Chem. Inf. Model. 2014, 54, 266-277.

(33) Ho, B. K.; Curmi, P. M. G. Twist and Shear in B-Sheets and BRibbons. J. Mol. Biol. 2002, 317, 291-308.

(34) Sreerama, N.; Woody, R. W. Poly (Pro) II Helixes in Globular Proteins: Identification and Circular Dichroic Analysis. Biochemistry 1994, 33, 10022-10025.

(35) Reed, J.; Reed, T. A. A Set of Constructed Type Spectra for the Practical Estimation of Peptide Secondary Structure from Circular Dichroism. Anal. Biochem. 1997, 254, 36-40.

(36) Leskovec, J.; Rajaraman, A.; Ullman, J. D. Mining of Massive Datasets, 2nd ed.; Cambridge University Press: Cameridge, 2014; Vol. 11.

(37) Mahrenholz, A.; Andersen, T. T.; Bao, Y.; Cohen, S. A.; Denslow, N. D.; Hulmes, J.; Hunziker, P. E.; Mann, K.; Schegg, K. M.; West, K. ABRF Amino Acid Analysis Survey: Identification of Proteins Electroblotted to PVDF, 1997.

(38) Gill, S. C.; Von Hippel, P. H. Calculation of Protein Extinction Coefficients from Amino Acid Sequence Data. Anal. Biochem. 1989, 182, 319-326.

(39) Anthis, N. J.; Clore, G. M. Sequence-Specific Determination of Protein and Peptide Concentrations by Absorbance at $205 \mathrm{Nm}$ : Sequence-Specific Protein Concentration at 205 Nm. Protein Sci. 2013, $22,851-858$.

(40) Lee, C. W.; Martinez-Yamout, M. A.; Dyson, H. J.; Wright, P. E. Structure of the p53 Transactivation Domain in Complex with the Nuclear Receptor Coactivator Binding Domain of CREB Binding Protein. Biochemistry 2010, 49, 9964-9971.

(41) Shen, Y.; Bax, A. SPARTA+: A Modest Improvement in Empirical NMR Chemical Shift Prediction by Means of an Artificial Neural Network. J. Biomol. NMR 2010, 48, 13-22. 